\title{
Erl1, a Novel Era-Like GTPase from Magnaporthe oryzae, Is Required for Full Root Virulence and Is Conserved in the Mutualistic Symbiont Glomus intraradices
}

\author{
Stephanie Heupel, ${ }^{1}$ Birgit Roser, ${ }^{1}$ Hannah Kuhn, ${ }^{1}$ Marc-Henri Lebrun, ${ }^{2}$ Francois Villalba, ${ }^{3}$ and \\ Natalia Requena ${ }^{1}$ \\ ${ }^{1}$ Plant-Microbe Interactions, Botanical Institute, University of Karlsruhe and Karlsruhe Institute of Technology (KIT), \\ Hertzstrasse 16, D-76187 Karlsruhe, Germany; ${ }^{2}$ UMR2847 Centre National de la Recherche Scientifique/Bayer Crop \\ Science, 14 Rue Pierre Baizet, 69263 Lyon Cedex 09, France; ${ }^{3}$ Biochemistry, Bayer Crop Science, Lyon, France
}

Submitted 9 June 2009. Accepted 27 August 2009.

Comparative analyses of genome sequences from several plant-infecting fungi have shown conservation and expansion of protein families with plant disease-related functions. Here, we show that this hypothesis can be extended to mutualistic symbiotic fungi. We have identified a gene encoding an Era (Escherichia coli Ras)-like GTPase in the rice blast fungus Magnaporthe oryzae and found that it is orthologous to the mature amino terminal part of the Gin1 protein from the arbuscular mycorrhizal (AM) fungus Glomus intraradices. M. oryzae Erl1 is required for full root virulence. Appressoria formation was not severely affected in Derllstrains, but invasive hyphae grew slower than in the wild type. Root browning defect of $\Delta$ erl1 strains could be complemented by the AM gene under the control of the ERL1 promoter. Erl1 and Gin-N localized to the nucleus when carboxy-terminally labeled with green fluorescent protein (GFP). However, amino-terminal GFP-tagged versions of the proteins expressed in Aspergillus nidulans were shown to localize in the cytoplasm and to cause polarity defects. These data suggest that Erl1 and Gin-N are orthologs and might be involved in the control of hyphal growth in planta. This is the first characterization of an Era-like GTPase in filamentous fungi.

The Magnaporthe grisea species complex is one of the most important groups of fungal pathogens threatening economically relevant cereals and grasses such as rice or wheat (Talbot 2003). The ascomycetous fungus Magnaporthe oryzae belongs to that group. The biology of the fungal-plant association of Magnaporthe oryzae and its host plant rice has been thoroughly studied, and $M$. oryzae is well known for its ability to mechanically breach the outer plant leaf surface. On the leaf surface, the $M$. oryzae germ tube morphologically differentiates upon recognition of physico-chemical cues into an appressorium, a dome-shaped melanized structure separated from the germ tube by a septum. Penetration is mainly mechanical, by generation of an enormous turgor pressure due to accumulation of glycerol up to $3.2 \mathrm{M}$ (de Jong et al. 1997). After penetration, a bulbous invasive hypha develops into the host cell

Corresponding author: N. Requena: E-mail: Natalia.requena@bio.uka.de; Telephone:. +49 (0)721 6084626; Fax: +49 (0)721 6084509.

* The $e$-Xtra logo stands for "electronic extra" and indicates that a supplementary table is published online. Figures 1 to $4,6,7$, and 9 appear in color online. and fills its lumen before proceeding to the neighbor plant cell through plasmodesmata (Kankanala et al. 2007). M. oryzae is considered a hemibiotroph because, during a long part of its infection cycle, the fungus maintains the plant alive to feed on living cells (Talbot 2003). Only in later stages does M. oryzae behave as a necrotroph, producing a series of toxic products that induce host cell death and cause the appearance of the typical leaf lesions. During its biotrophic phase, M. oryzae does not break the plasma membrane of the invaded host cells and infection hyphae are continuously tightly surrounded by the host plasma membrane, similarly to other biotrophic interactions (Kankanala et al. 2007). How is the biotrophic status established and maintained in compatible interactions? The comparative analysis of fungal as well as oomycete plant-infection strategies has shed light on this fascinating aspect, showing that an arsenal of secreted effectors that enter the plant cytoplasm are responsible for defeating plant cell defenses (Soanes et al. 2008). Indeed, genome analyses of different fungi that interact with plants, including mutualistic symbionts, have shown that all do possess extensive families of secreted proteins of unknown function that might perform as putative effectors (Dean et al. 2005; Kämper et al. 2006; Martin et al. 2008). Recent transcriptome analyses of $M$. oryzae during the biotrophic phase has led to the identification of genes encoding potential secreted effector proteins that are highly induced during the in planta phase (Mosquera et al. 2009).

The pathogenic cycle of $M$. oryzae predominantly develops in leaves; very recently however, it was recognized that $M$. oryzae is also capable of infecting roots and spreading from there to the shoot (Dufresne and Osbourn 2001). The analysis of the infection cycle through the root showed that the fungus does not require melanized appressoria to colonize this tissue (Sesma and Osbourn 2004). Instead, the fungus develops the so-called hyphopodia. These structures are small swellings at the hyphal tip, nonmelanized and not separated by a septum. Hypophodia are common in root-infecting fungi, including arbuscular mycorrhizal (AM) fungi that form symbiotic relationships with plant roots (Genre et al. 2009). The lack of a septum behind the hyphopodia and the absence of melanin in this structure indicate that turgor pressure is not the major force required to penetrate the root. This is not surprising, considering that the surface of the rhizodermis is not so hard and hydrophobic as the leaf cuticula. However, it opens the question of whether the fungus recruits the root cellular machinery to penetrate, as in mutualistic interactions (Genre et al. 2008), produces a cocktail of cell wall-degrading enzymes, or both. 
The differential morphogenesis between hyphopodia and appressoria is linked to a genetic program acting in roots vs. leaves that is induced on the basis of different hydrophobicity and possibly other physico-chemical cues. Thus, while melanin production and cAMP signaling are essential for appressoria formation, they are dispensable for root colonization (Dufresne and Osbourn 2001; Sesma and Osbourn 2004). Likewise, NUT1, a homolog of AreA, a global regulator of nitrogen metabolism from $A$. nidulans, is only required for root infection (Dufresne and Osbourn 2001). More interestingly, $M$. oryzae shares some of the genetic components specific for root colonization with other root-infecting fungi (Sesma and Osbourn 2004). A homolog of the Fusarium oxysporum FOWI gene, a mitochondrial protein required for tomato-root colonization (Inoue et al. 2002) was found to be also required for full virulence at the root of $M$. oryzae. Mutants lacking FOWI arrest within the second cell layer (Sesma and Osbourn 2004), however, they are unaffected in appressoria formation on artificial surfaces and in pathogenicity to leaves. From these results, it is possible to reason that mechanisms and the responsible genes required for root colonization may be conserved among soil-borne fungi, including mutualistic symbionts like AM fungi. This could, in turn, explain why plants react with partially overlapping responses to such different colonizing fungi such as $G$. intraradices, $M$. oryzae, or Fusarium moniliforme (Güimil et al. 2005).

Our goal is to investigate whether mutualistic symbiotic fungi such as $G$. intraradices and pathogenic fungi such as $M$. oryzae might use a conserved pathway for root infection, in which orthologous proteins might play dual roles. We obtained evidence for this hypothesis from the work described in this paper. It is based on the AM Gin1 (GTPase, intein) protein, identified by our group, that is presumed to play a role in establishing compatibility with the plant. Gin1 is a two-domain protein identified in a screening for genes expressed during the early stages of mycorrhiza formation (Requena et al. 2002, 2007). The amino terminus has homology to Ras-like GTPases while the carboxy terminus contains the Hint domain (Hedgehog/intein) necessary for protein autoprocessing of hedgehog proteins (Paulus 2000). We have shown that Gin1 can be posttranslationally cleaved in vitro by plant signals, to render the mature amino terminus (Requena et al. 2007; E. Serrano and N. Requena, unpublished results). In this work, we have identified a putative ortholog of the amino terminus of the G. intraradices Gin1 in $M$. oryzae. The gene encodes a novel GTPase from the Era (E. coli-Ras)-like family of GTPases that we named Erl1. We show here that $M$. oryzae Erl1 was dispensable for saprophytic growth, appressoria formation, or production of leaf lesions, although it reduced the growth of invasive hyphae. However, deletion of ERL1 significantly reduced root browning when $M$. oryzae was inoculated on the root. $G$. intraradices $G I N-N$ complemented the defect of ERL1 deletion in root disease when expressed under the ERL1 promoter. Both proteins localized to the nucleus and caused defects in polarized growth if mislocalized at the cytoplasm. Taken together, our results suggest that Gin-N and Erl1 are orthologs, supporting the hypothesis that symbiotic and pathogenic fungi use some conserved mechanisms for root infection.

\section{RESULTS}

\section{M. oryzae Erl1 is the closest fungal relative of $G$. intraradices Gin-N.}

We identified GIN1 in the fungus G. mosseae during a screening for genes involved in the early stages of the AM symbiosis. GIN1 encodes a novel protein resulting from the fusion of two domains (Requena et al. 2002). The amino ter- minus shows homology to several GTPases, while the carboxy terminus contains the Hint domain necessary for protein autoprocessing typical of hedgehog proteins (Paulus 2000). We have further identified the ortholog of GIN1 in the model AM fungus $G$. intraradices and have shown that the encoded protein is post-translationally modified to render a free amino terminus (Requena et al. 2007; E. Serrano and N. Requena, unpublished results). The amino terminus of $G$. intraradices Gin1(Gin-N) comprises 237 amino acids. Domain analysis in the National Center for Biotechnology Information (NCBI) database has shown that Gin-N contains the conserved domain characteristic of proteins from the Ras superfamily of GTPases. This is a large group of proteins, including many different families. Gin-N encompassed the conserved domains cd01852 and cd00880 from the AIG1 (avirulence-induced gene) and Eralike families, respectively (Marchler-Bauer et al. 2007). Although, Gin-N is more closely related to animal than to fungal proteins, the closest fungal homologs of Gin-N are the hypothetical proteins MGG_02549 from $M$. oryzae (formerly $M$. grisea 70-15) and $\mathrm{CC}_{1 \mathrm{G}} 12375$ from Coprinopsis cinerea, both containing the Era-like signature. The Era-like protein family includes several distinct subfamilies, with several representative members in bacteria, such as TrmE, YihA, EngA, and Era, that regulate diverse cellular functions including ribosome function, cell cycle, DNA partitioning, and DNA segregation (Caldon and March 2003). Proteins from the Era-like family are poorly defined and, according to the Conserved Domains database in NCBI, are characterized by a lack or poor conservation of the $\mathrm{G} 4$ and $\mathrm{G} 5$ boxes. ClustalW alignment of Gin-N, MGG_02549, andCC1G_12375, together with the closest animal sequence belonging to the AIG1 subfamily (HIMAP4) revealed that the fungal proteins all lacked apparent G4 and G5 boxes. Thus, it appears that these fungal proteins might rather be more related to the Era-like GTPase family than to the AIG family. The conservation among the fungal proteins is almost exclusively restricted to the amino terminus, while the carboxy terminus is highly variable (Fig. 1). We named the $M$. oryzae protein MGG_02549 as Erl1 (for Era-like).

\section{Deletion of ERL1 in M. oryzae does not affect growth but delays appressoria formation on less inductive surfaces.}

Knowledge about Era-like proteins in eukaryotes and, in particular, in fungi is scarce. While bacterial members have been more thoroughly investigated, only Mss1 from Saccharomyces cerevisiae, a homolog of the bacterial TrmE, has been characterized and shown to be involved in mitochondrial translation (Colby et al. 1998; Decoster et al. 1993). In order to characterize Erl1 in $M$. oryzae, we generated a deletion construct containing the upstream and downstream regions of the Erl1 coding sequence flanking the BASTA selection cassette (Sweigard et al. 1997) (Fig. 2A). Transformants of wild-type $M$. oryzae were analyzed by polymerase chain reaction (PCR) and Southern blot (Fig. 2B and C). Two different deletion strains (KO18 and KO20) were chosen for further analysis. We first analyzed the effect of ERL1 deletion on growth, conidiation, and appressoria formation on hydrophobic surfaces. Deletion strains KO18 and KO20 did not display any growth phenotype, including hyphal polarity, on complete medium (Fig. 3A). Both strains produced the same number of conidia as the wild-type strain, and the conidia did not show any morphological alteration (Fig. 3B). Appressoria formation on highly hydrophobic surfaces such as bioFOIL was not significantly different between Guy 11 and the Aerll strains after $16 \mathrm{~h}$ (Fig. $3 \mathrm{C}$ and D). However, we noticed that if conidia were deposited on less hydrophobic surfaces such as parafilm (compared with bioFOIL), appressoria formation was slightly delayed. After $16 \mathrm{~h}$, only $60 \%$ of conidia from deletion strains had formed 
appressoria, compared with $80 \%$ from the wild-type strain (Fig. 3E). In a time-course analysis on bioFOIL, deletion strains formed appressoria more slowly initially than did the wildtype strain, but this difference was no longer evident after $16 \mathrm{~h}$ (Fig. 3F).

\section{The ability to produce leaf disease symptoms is not compromised in $\Delta$ erl1 strains but in planta growth is slower.}

To test the ability of $M$. oryzae Aerll to form appressoria and to colonize plant cells, we next performed the onion epidermis penetration assay (Bhambra et al. 2006) with green fluorescent protein (GFP)-tagged strains. Wild-type and deletion strains were transformed with a construct constitutively expressing GFP under the control of the ToxA promoter (Sesma and Osbourn 2004). The $\Delta e r l 1$ strain was able to form appressoria and to colonize onion cells intracellularly (Fig. 4A; only $\mathrm{KO} 20$ is shown, results from strain KO18 were comparable). However, although the number of appressoria after $24 \mathrm{~h}$ and the number of infected cells was similar between both strains (left and middle panels), the deletion strain developed more slowly in planta and showed less intracellular colonization after $48 \mathrm{~h}$ (middle and right panels). To investigate whether this delay in cellular colonization was reflected in the number or size of necrotic lesions produced, rice leaves were surfaceinoculated with spore suspensions of wild type or the Aerll strain. After 5 days, all leaves showed similar lesions (Fig. 4B). The same was observed on abraded rice leaves from which the cuticula had been removed (Fig. 4C). This result indicates that, although growth of biotrophic hyphae is delayed in $\Delta e r l 1$, the necrotic ability of the strain is not compromised.

\section{Erl1 is required for full root virulence.}

Given that Erll was identified as a putative ortholog of the $G$. intraradices Gin- $N$ and that $G$. intraradices exclusively colonizes plant roots, we next asked whether deletion of ERL1 would impair the ability of $M$. oryzae to infect roots. Rice seedlings were inoculated with fungal plugs of the wild type and of Aerll strains as described (Dufresne and Osbourn 2001). Interestingly, Aerll strains were less virulent than Guy11. Although plants inoculated with the deletion strains also showed necrotic symptoms after 15 days, significantly less browning was produced compared with the wild type (Fig. 5A; Table 1). To assess whether the reduced virulence of $\Delta e r l 1$ in roots could be due to impairment in hyphopodia formation or rhizodermis penetration, we tagged the wild type and the deletion strain KO20 with GFP. However, although the Aerll-GFPtagged strain also showed less root browning than Guy11-GFP (Fig. 5B; Table 1), both strains were able to form hyphopodia on the root surface, penetrate the rhizodermis, and colonize intracellularly the root cortex cells (Fig. 5C).

\section{G. intraradices Gin- $N$ complements \\ the root virulence defect of $M$. oryzae $\Delta e r l 1$.}

To prove that the phenotype observed was due to the deletion of ERL1, we retransformed the mutant strains using a cosmid containing the complete genomic sequence of ERL1. Recomplemented strains were used to infect both leaves and roots of rice. All strains produced a similar number of lesions on rice leaves (Fig. 6A). In contrast, roots were significantly less necrotic when inoculated with deletion strains compared with plants inoculated with wild type or recomplemented strains (Fig. 6B). While the 9N9/18 recomplemented strain showed a full restoration of the necrotic effect, strain 9N9/20 showed only partial restoration (Table 1). Ectopic expression of Erl1 tagged with GFP also partially restored the root browning defect of the deletion strains (Fig. 5B; Table 1). Therefore, the less virulent phenotype observed in Aerll strains can be attributed to the deletion of the corresponding gene. In order to test whether $M$. oryzae Erl1 and $G$. intraradices Gin-N are true orthologs, the coding sequence of Erl1 was replaced in locus by $G I N-N$ to be expressed under the control of the ERLI promoter. Two strains containing this gene arrangement were produced. One strain was obtained through homologous recombination after transformation of the wild-type strain. The second strain was generated by in locus replacement of the BASTA cassette of the KO18 strain. Growth phenotype under different selection media and Southern blot analyses proved that both strains contained the $G I N-N$ gene in the locus of ERLI (Fig. 7A and B). Wild type, deletion strain (KO18), and the strain expressing $G I N-N$ from the ERL1 promoter [ERL1::GIN-N (1)] were used to inoculate rice roots. As shown in Figure $7 \mathrm{C}$ and Table 1, the $M$. oryzae ERL1::GIN-N (1) strain showed higher rates of disease symptoms than did the $\Delta e r l 1$ strains, albeit not

\begin{abstract}
GiGin-8 MOEr11

Fig. 1. Protein alignment of three putative filamentous fungal Era (Escherichia coli Ras)-like proteins. ClustalW multiple alignment showing the two closest fungal and the closest animal sequences to the Glomus intraradices Gin-N protein (Thompson et al. 1994). The G boxes from G1 to G5 are boxed. Switch regions I and II are marked with a dark gray bar while the GTP/Mg ${ }^{2+}$ binding site is marked with a light gray bar. Accession numbers: Magnaporthe oryzae Erl1 (XP_366473), Coprinopsis cinerea (XP_001828348), and Homo sapiens HIMAP4 (EAL 24482).
\end{abstract}


as high as rates in the wild-type strain. This result indicates that $G$. intraradices Gin-N can partially complement $M$. oryzae Erl1 function and, thus, it might be a true ortholog.

\section{Erl1 and Gin-N localize to the nucleus.}

To our knowledge, Erl1 is the first member from the Eralike subfamily of GTPases to be characterized in filamentous fungi. In order to gather information about the function of this protein, we investigated its subcellular localization. The protein was tagged with GFP at the carboxy terminus and was expressed in $M$. oryzae Guy11 under the control of the $A$. nidulans alcA promoter. We choose to use this heterologous promoter because the rate of expression of natural promoter is very low in vegetative hyphae (data not shown). The alcA promoter has been reported to work in Neurospora crassa as well as in planta (Roslan et al. 2001; Wang and Keasling 2002). In A. nidulans it can be repressed by glucose, derepressed in glycerol, and induced by threonine or ethanol. Fluorescence microscopy analyses of transformed $M$. oryzae showed that Erl1-GFP protein was mainly targeted to the nucleus (Fig. 8A and B). However, a high level of fluorescence was always observed, even on glucose-containing media, and the protein could also be observed in other subcellular localizations, like endoplasmatic reticulum projections towards the nucleus at the appressorium (Fig. 8B and C). This showed that the alcA promoter functions constitutively in $M$. oryzae and it is not subjected to catabolite repression. In order to better visualize the subcellular localization of Erl1-GFP, the same construct as well as the fusion Gin-N-GFP, also under the control of the alcA promoter, were expressed in A. nidulans. In this system, both proteins Erl1-GFP and Gin-N-GFP showed a clear and exclusive nuclear localization pattern, both in glycerol and in threoninecontaining media (Fig. 8D). This was somewhat surprising, because none of the proteins contains a clear nuclear targeting

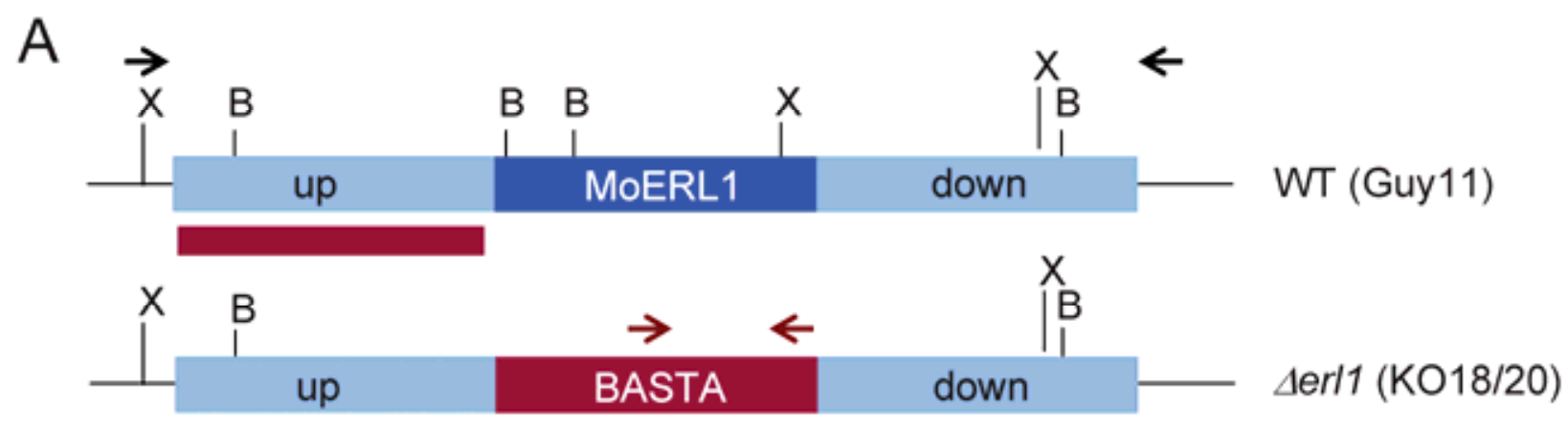

B

PCR UPKO: ca. $2 \mathrm{~Kb}$

$171820 \mathrm{~N}$

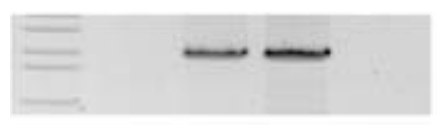

PCR DOWNKO: $1.6 \mathrm{~Kb}$

$\begin{array}{llll}17 & 18 & 20 & \mathrm{~N}\end{array}$

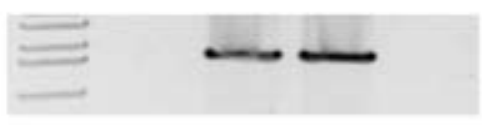

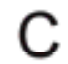
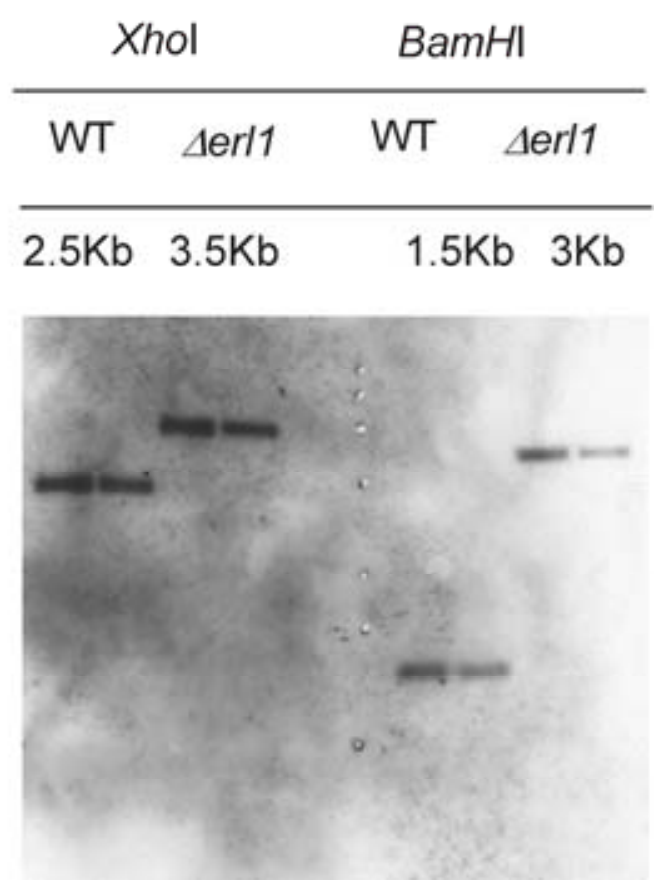

Fig. 2. Deletion of Magnaporthe oryzae ERL1. A, Physical map of the M. oryzae ERL1 wild-type genomic region and of the deletion strains after homologous integration of the replacement cassette containing the BASTA (Sweigard et al. 1997) resistance gene. Restriction enzyme sites for BamHI (B) and for XhoI (X) are indicated. Arrows represent the approximate position of oligonucleotides used for polymerase chain reaction (PCR) amplification to screen the mutant strains. B, Results of the PCR amplification on three transformants, showing that only two of them contain the replacement cassette inserted in the ERL1 locus. C, Autoradiograph of a Southern blot using deletion strains KO18 and KO20. Genomic DNA of two wild-type strains and the two mutant strains digested with $\mathrm{XhoI}$ and BamHI was probed with the upstream region of ERL1 indicated in A. Deletion strains showed the expected shift in size. 

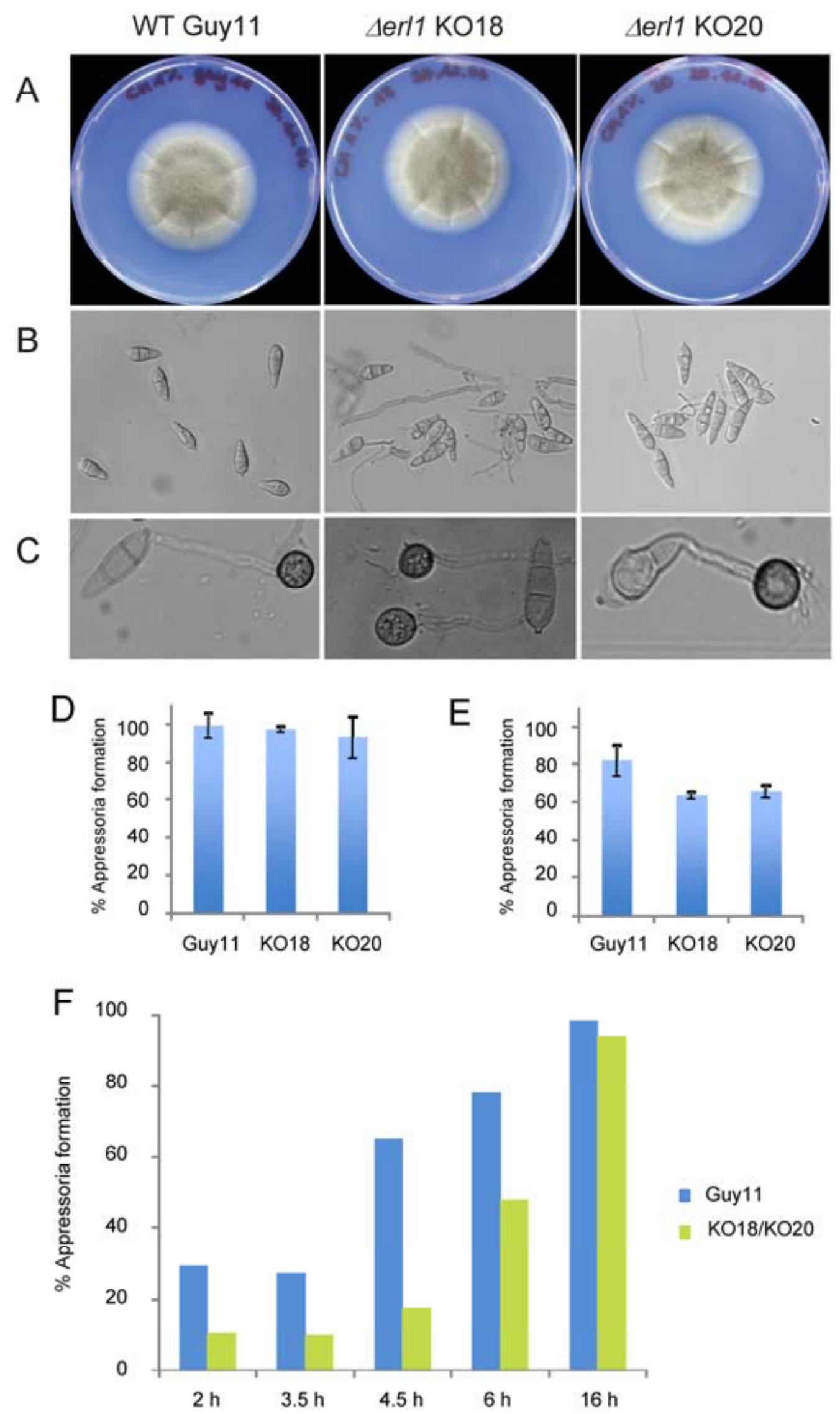

Fig. 3. Erl1 is dispensable for growth, sporulation, and appressoria formation. A, ERL1 deletion strains KO18 and KO20 did not show any difference with respect to the wild-type strain when growing on artificial substrates, as shown here on complete medium. $\mathbf{B}$, Conidiation was identical among the three strains and $\mathbf{C}$ and D, appressoria formation on bioFOIL after $16 \mathrm{~h}$ was also not different. E, Appressoria formation on less hydrophobic surfaces such as Parafilm was slightly reduced in ERL1 deletion strains. F, Timecourse of appressoria formation on bioFOIL showed that ERL1 deletion strains were initially slower than the wild-type Guy11 strain, but this difference was no longer visible after $16 \mathrm{~h}$. The $y$ axis in $\mathrm{D}, \mathrm{E}$, and F represents percentage of appressoria formation. 
signal. Since Gin-N is produced in $G$. intraradices as a preprotein containing a longer carboxy terminus that is post-translationally cleaved, we expressed the full-length Gin1 protein (containing the Hint domain) carboxy-terminally labeled with GFP. The full-length Gin1 protein was also targeted to the nucleus, showing that, in $A$. nidulans, the autoprocessing of the carboxy terminus does not take place and it is not required for nuclear localization (Fig. 8D). Interestingly, when GFP was fused to the amino terminus of any of the three proteins, they were no longer targeted to the nucleus but to the cytoplasm (Fig. 9A). This indicates that a nuclear localization signal or a domain for interacting with proteins that shuttle them into the nucleus is possibly located at or close to the amino terminus of the Era-like fungal proteins. The presence of GFP in this location could interfere with that signal, resulting in cytoplasmatic protein localization. Most interestingly, while the expression of the three proteins with a GFP tag at the carboxy terminus showed a normal growth phenotype in all media tested, strains expressing proteins fused at the amino terminus exhibited an aberrant hyphal phenotype in which polarized growth was affected. These hyphae grew no longer straight but curled and, in contrast to the wild-type strain, with dichotomous branching,
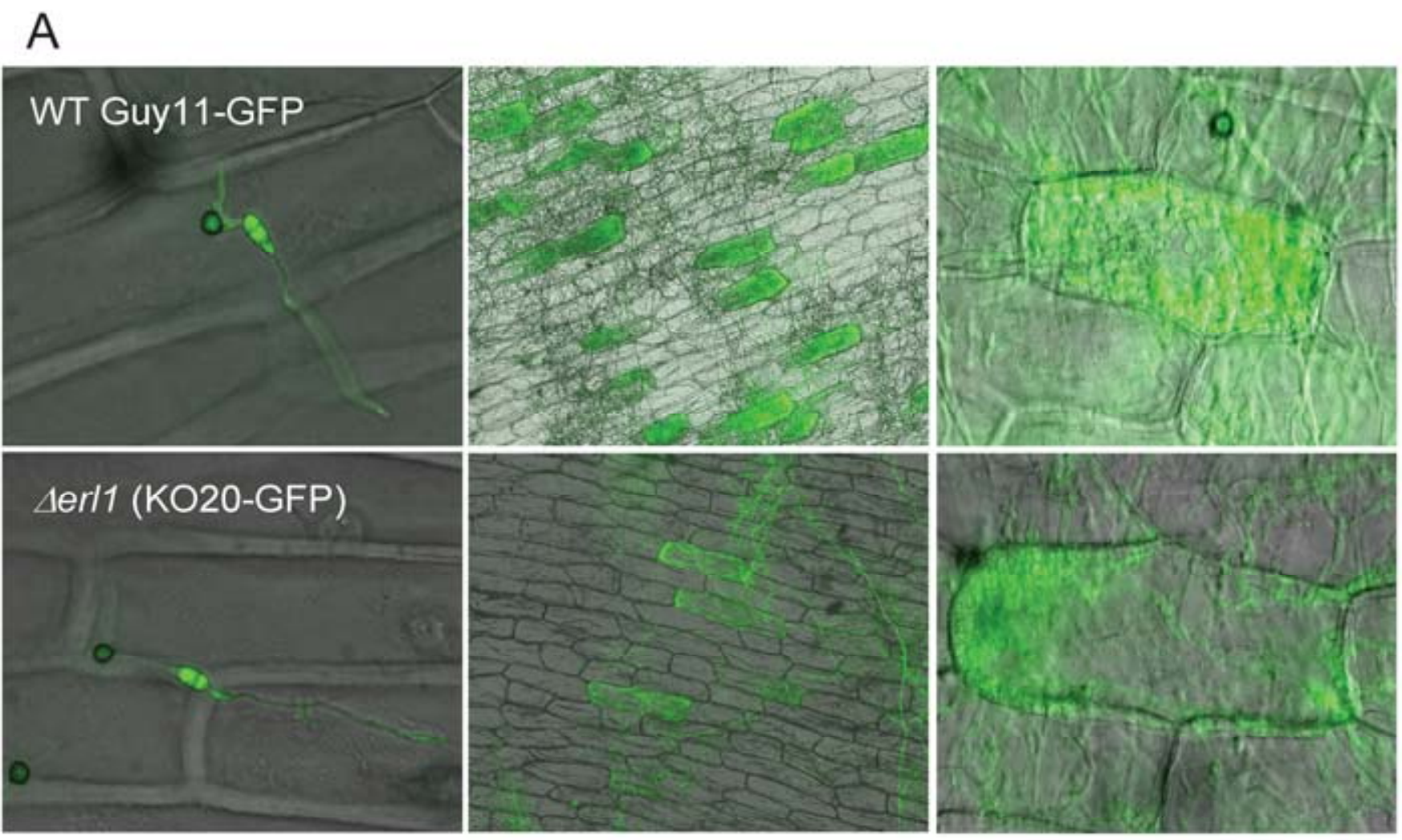

\section{B}

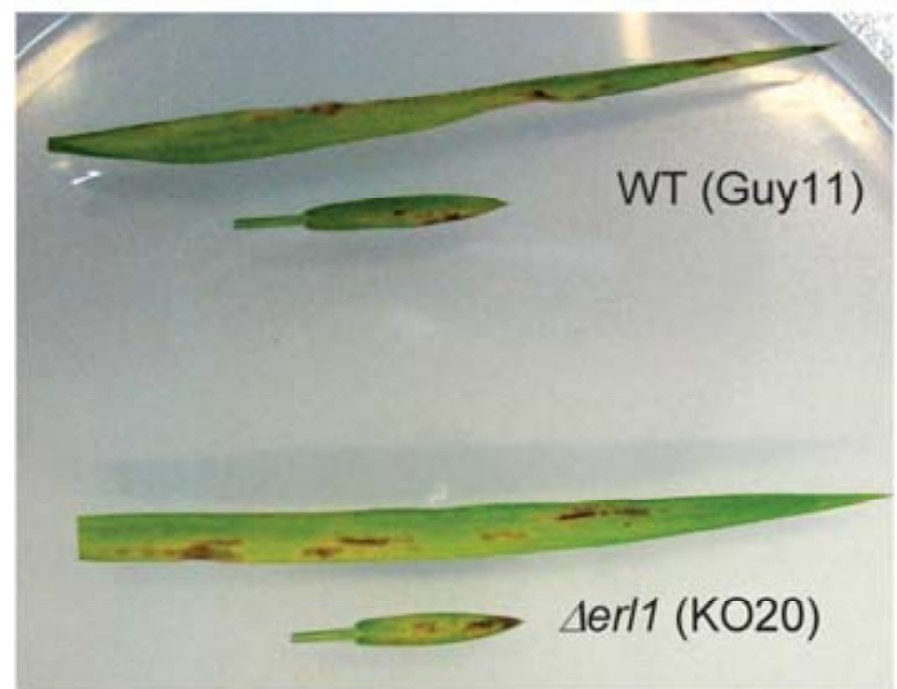

C

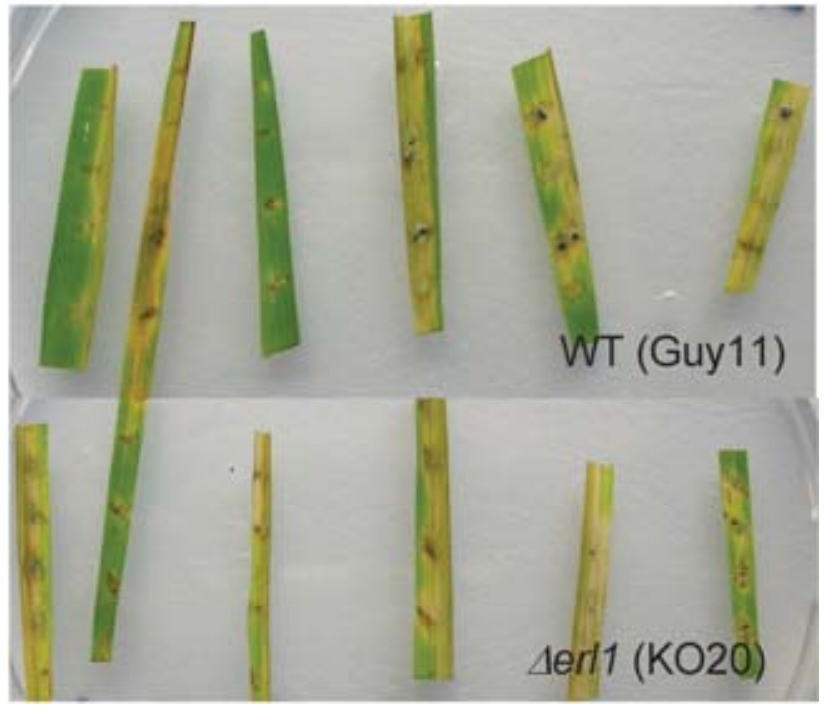

Fig. 4. Leaf colonization and pathogenic capability of $\Delta e r l 1$ strains. A, The onion epidermis assay was employed to analyze the ability of $\Delta e r l 1$ strains to form appressoria and colonize leaf cells. Conidia suspensions were used to inoculate onion epidermal cells. Appressoria formation observed after $24 \mathrm{~h}$ was identical between the Guy11 and KO20 strains. However, when the samples were observed after $48 \mathrm{~h}$, a delay in colonization of the cell lumen was observed in the KO20 strain as compared with the wild-type strain. $\mathbf{B}$ and $\mathbf{C}$, No differences were observed between Guy11 and KO20 strains in the number or degree of lessions produced by conidia spray-inoculation on intact leaves (B) nor after conidia inoculation onto abraded leaves (C). 

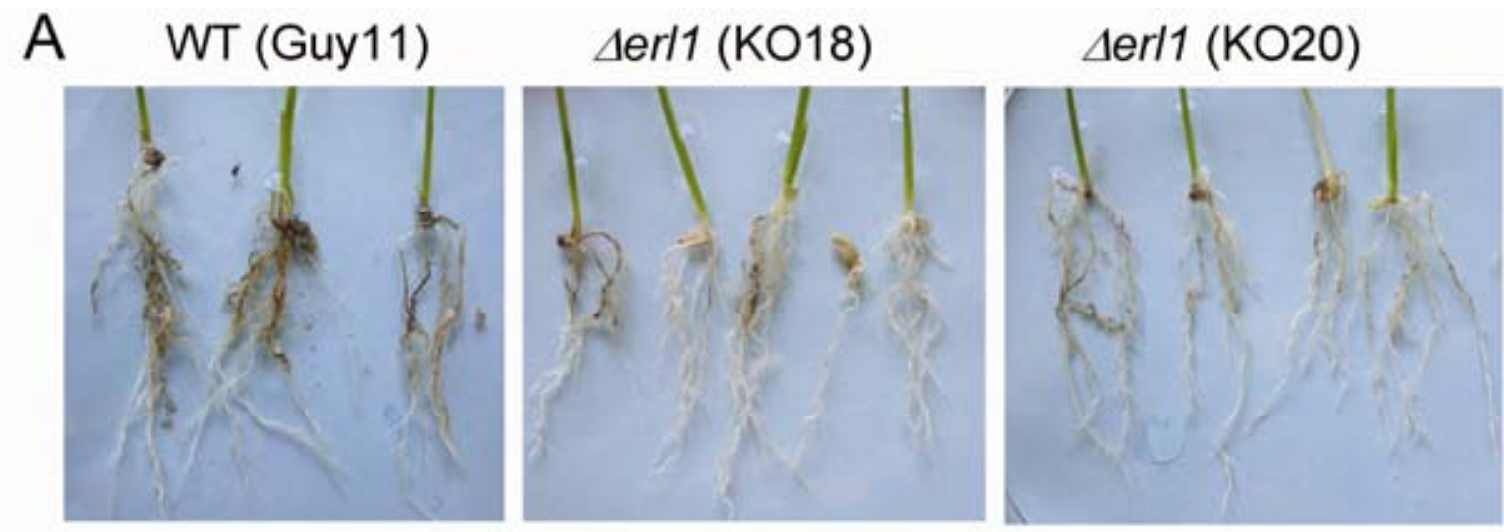

B
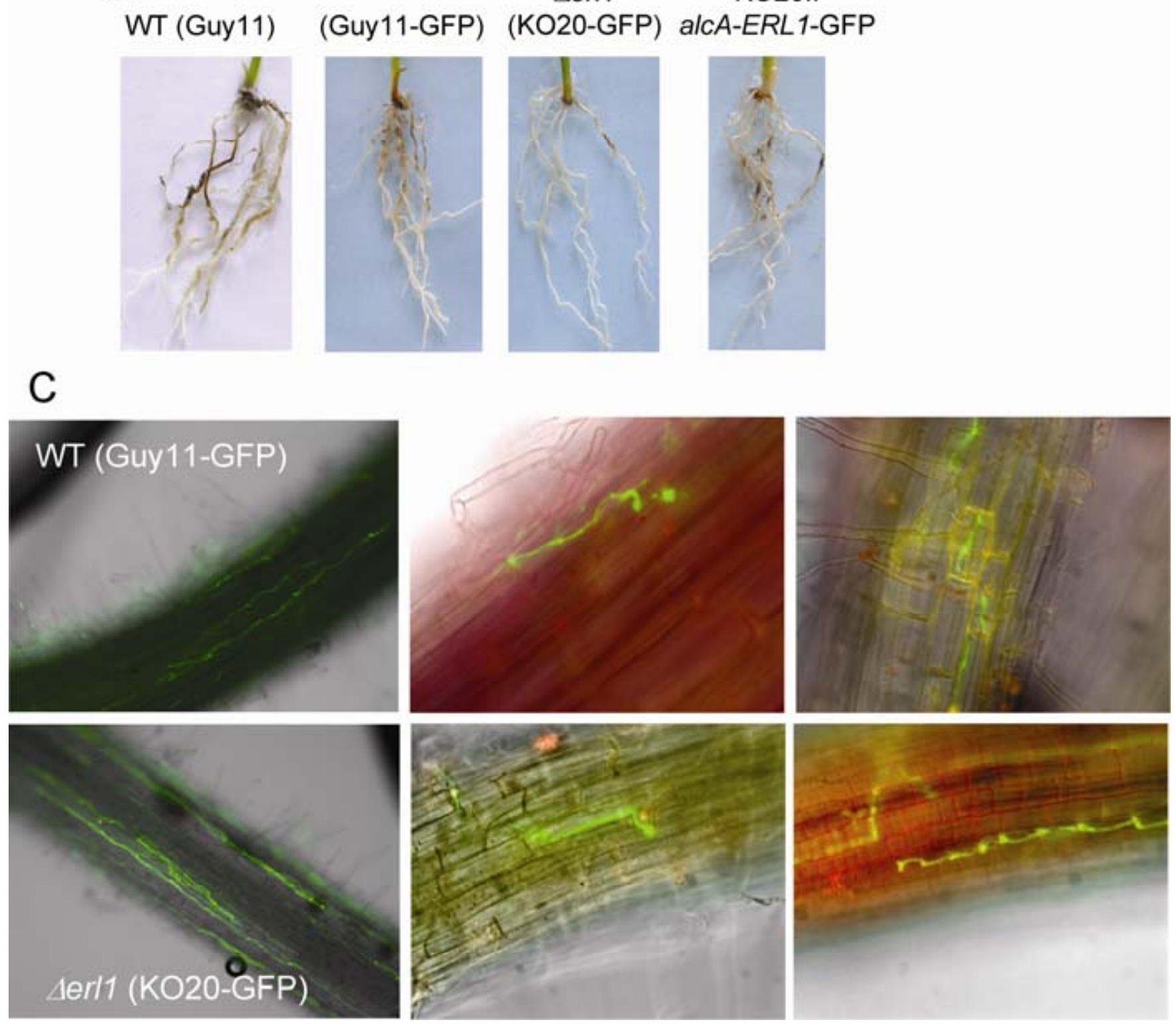

Fig. 5. Root browning is reduced in $\Delta e r l 1$ strains. A, Ability of $\Delta e r l 1$ strains KO18 and KO20 to induce root browning was assessed on rice cultures 15 days after inoculation, using mycelial plugs. Deletion strains showed a marked decrease in necrotic symptoms compared with the wild type. B, Root symptoms produced by wild type and the KO20 deletion strain labeled with green fluorescent protein (GFP) compared with the unlabeled wild type. For comparison, the ectopic expression of Erl1-GFP in the deletion strain background of KO20 is shown. C, GFP-labeled wild type and the KO20 deletion strain were used to monitor hyphopodia and intracellular root colonization. Both strains were able to produce hyphopodia and colonize intercellularly the root cortex. 
and bipolar germination was often observed (Fig. 9B). The curled phenotype is more pronounced in threonine-containing media than on glycerol and not visible at all on glucose. This means that the amount of protein in the cytoplasm is what determines the aberrant growth phenotype.

\section{DISCUSSION}

The rapidly increasing number of sequenced genomes has allowed a thorough comparative analysis to dissect the mechanisms and weapons used by diverse pathogenic fungi during infection of their host plants. It became evident that several of the strategies are conserved among different fungal pathogens and that parts of the components required for infection are present in fungi infecting completely different hosts. Although in some cases, this could be attributed to a convergent evolution, in other cases, it possibly reflects the maintenance of a successful tool during fungal evolution (Soanes et al. 2008). In this work, we present evidence that the latter might be also the case of a fungal protein that is required for full virulence in root infections in the blast fungus $M$. oryzae and that is conserved in the mutualistic symbiont $G$. intraradices.

AM fungi are the biotrophs per excellence. They maintain long-term relationships with their hosts that last the whole life span of the plant (Genre et al. 2009). After penetration of the rhizoderm using hyphopodia, AM fungi colonize the root cortex inter- and intracellularly but never penetrate the host plasma membrane. In inner cortical cells, AM fungi develop arbuscules, highly branched hyphal ramifications that are similar to haustoria. The discovery that $M$. oryzae can colonize roots using hyphopodia instead of appressoria, similar to other root-infecting fungi, the fact that the initial $M$. oryzae biotrophic phase is also operational in roots, and the finding that rice roots react partially similar to both fungi prompted us to investigate whether AM fungi and M. oryzae could share some of the machinery required to colonize the root. In this work, we concentrated on Erl1, the putative ortholog of the AM protein Gin1, identified by sequence similarity and motif conservation in M. oryzae. ERL1 encodes a GTPase of the RAS-like superfamily that shares with Gin 1 the Era-like signature. Era was found as the first example of a prokaryotic gene with homology to the yeast Ras protein (Ahnn et al. 1986). However, a closer analysis revealed that this homology was mainly restricted to the amino terminus. Nevertheless, homologs of Era were subsequently found in several eukaryotes, including humans, and were found to bind RNA and to play an important role in the control of apoptosis (Akiyama et al. 2001). Later on, with the outcome of new genome sequences, several new GTPases of unknown function, but with some similarity to Era proteins were found, both in prokaryotes and eukaryotes, and they were generally named as Era-like GTPases (Mittenhuber

Table 1. Evaluation of root browning in Nipponbare rice roots 15 days after inoculation

\begin{tabular}{lc}
\hline Medicago oryzae strains & Root browning $(\boldsymbol{\%})^{\mathbf{b}}$ \\
\hline Guy11 & $81.5 \pm 5.9$ \\
KO18 & $30.4 \pm 12.2$ \\
KO20 & $16.1 \pm 0.8$ \\
9N9/KO18 & $73.3 \pm 15.8$ \\
9N9/KO20 & $47.0 \pm 13.0$ \\
Guy11-GFP & $65.0 \pm 9.3$ \\
Aerll-GFPa & $22.5 \pm 4.6$ \\
Aerll::ERL-GFP & $51.3 \pm 9.9$ \\
ERL1::GIN-N & $51.7 \pm 9.8$
\end{tabular}

${ }^{\mathrm{a}} \mathrm{GFP}=$ green fluorescent proteion; ERL = Era-like; GIN = GTPase intein

${ }^{b}$ Values indicate percentage of root browning (extension and intensity) \pm standard error among at least three biological replicates.
2001). However, with the exception of the conserved GTPase domain, the proteins differ significantly in their overall structure, and only a few of them have been characterized in detail (Cabedo et al. 1999; Gong et al. 2004). In eukaryotes, only $S$. cerevisiae Mss1p, a homolog of the $50 \mathrm{~K}$ protein of bacteria (TrmE or MnmE) has been studied in more detail (Colby et al. 1998; Decoster et al. 1993). Erl1 or Gin1 share little similarity to other Era-like proteins and seem to represent a new subfamily. We decided to analyze the role of Erl1 in M. oryzae.

We could show here that Erll is not required for growth on synthetic media. Speed of growth, polarity, and conidiation were not affected, and deletion strains behaved in that respect identical to the wild-type strain. Therefore, it appears that this GTPase might not have a role during the saprotrophic stage of $M$. oryzae. However, when the interaction with the plant was studied, $\Delta e r l l$ strains were found to be less virulent, especially towards the root. We observed that, while appressoria formation on highly hydrophobic surfaces such as bioFOIL or the leaf cuticula were not affected in Aerll strains, in less hydrophobic surfaces such as parafilm, a delay in appressoria formation occurred. Although it was not possible to quantify properly the number of hyphopodia formed on the rhizodermis by mycelia from $\Delta e r l 1$ and wild-type strains, it was evident that necrosis symptoms produced by the wild-type strain in roots were either earlier, stronger, or both. Taken together, these results suggest that Aerll strains were impaired in their development on less hydrophobic surfaces such as the root, which would delay the subsequent necrotic phase. Alternatively the switch from the biotrophic to the necrotrophic phase in Aerll strains could be affected. At the moment, it is not possible to decide which alternative is correct. However, the fact that growth of invading hyphae within leaf epidermal cells was reduced in $\Delta e r l 1$ strains suggests that Erl1 could rather be involved in the regulation of the biotrophic to necrotrophic switch.

The amino terminus of the G. intraradices Gin1, Gin-N, is able to complement the deletion of ERL1, at least partially restoring the virulence towards the root and suggesting that both proteins might be true orthologs. Thus, together with the ortholog of the FOWl gene from $F$. oxysporum found in $M$. oryzae, this is the second example of a conserved gene required for root colonization. Even more, this is the first example showing that the conservation extends further from pathogenic to mutualistic fungi.

Carboxy-terminally GFP-tagged versions of Erl1 and Gin-N were shown to localize to the nucleus, both in M. oryzae and in A. nidulans. In bacteria, the Era-like protein TrmE was shown to control the transcription and translation of components of the $\mathrm{pH}$ regulatory machinery (Gong et al. 2004). Although it is currently unclear whether the control is exerted directly or indirectly, the GTPase activity was shown to be essential for the observed control. It is very well possible that the localization of Erl1 and Gin1 in the nucleus may be related to a function in transcriptional control. Further studies using protein-protein interaction methods might help in deciphering the function of Erl1.

The amino-terminal labeling of Erl1 and Gin-N with GFP led, however, to a cytoplasmic localization of both proteins that resulted in severe defects on hyphal polarity. Given that these proteins are similar to other small GTPases, it is possible that, when wrongly localized in A. nidulans, they interfere with components required for polarity maintenance. Although we assume that this effect might be due to the mislocalization of the proteins, it is a further proof that both proteins are orthologs. We attribute the cytoplasmic localization to a possible steric interference of GFP with a nuclear localization signal located at the amino terminus of Erl1 and Gin-N proteins. It is also possible that nuclear localization takes place by a piggy- 
bag mechanism involving interaction with another protein. However, since we have not studied the dynamic localization of Erl1 during the $M$. oryzae life cycle, we cannot exclude the possibility that, under natural conditions, the protein will not always be located at the nucleus. Similar to other GTPases (Williams 2003), Erl1 might shuttle between subcellular compartments. Considering this possibility, the dramatic changes observed in A. nidulans polarity caused by cytoplasmic localization of Erl1 or Gin-N might not represent artifacts but may give a hint of the true function of the protein.

Small GTPases are essential protein switches controlling many aspects of fungal cell differentiation and development, including pathogenicity (Chen et al. 2006; Mahlert et al. 2006; Zheng et al. 2007). Recently, they have been shown to be the
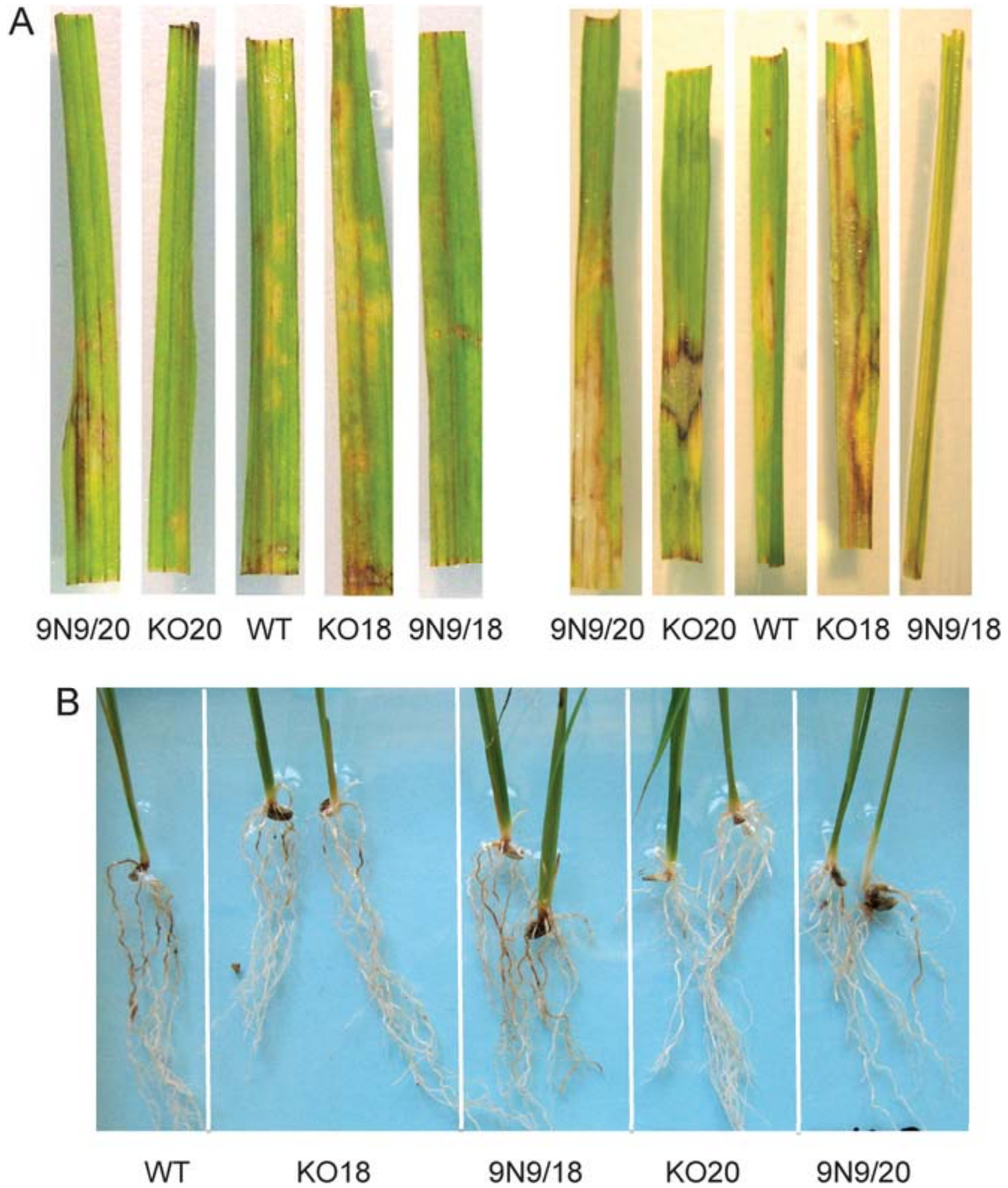

Fig. 6. ERL1 is responsible for the less-browning root phenotype of $\Delta e r l 1$ strains. $\Delta e r l 1$ strains KO18 and KO20 were recomplemented, using the cosmid 9N9 containing the genomic region in which ERL1 is located. A, Leaf infection ability of wild-type, deletion, and recomplemented strains. As expected, no difference was observed in the blast symptoms produced on leaves by the different strains analyzed. B, Root-browning defect of $\Delta e r l 1$ strains was restored when $E R L 1$ was ectopically integrated, confirming that the reduced root-browning phenotype is due to the absence of ERL1. 
A

$\mathrm{CM}$

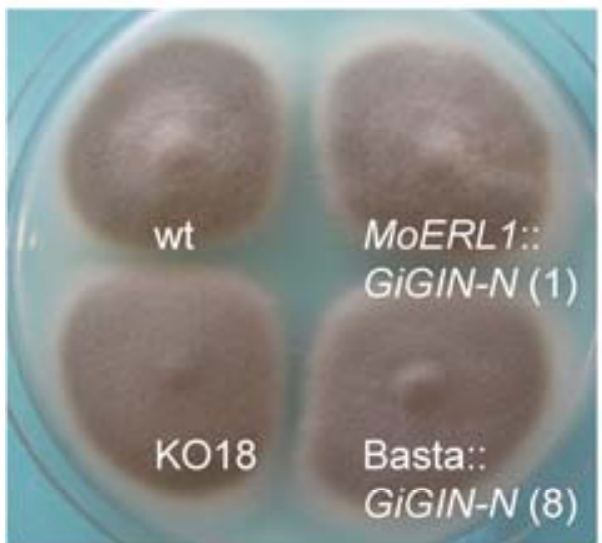

SW-Basta

(40 mg/l)

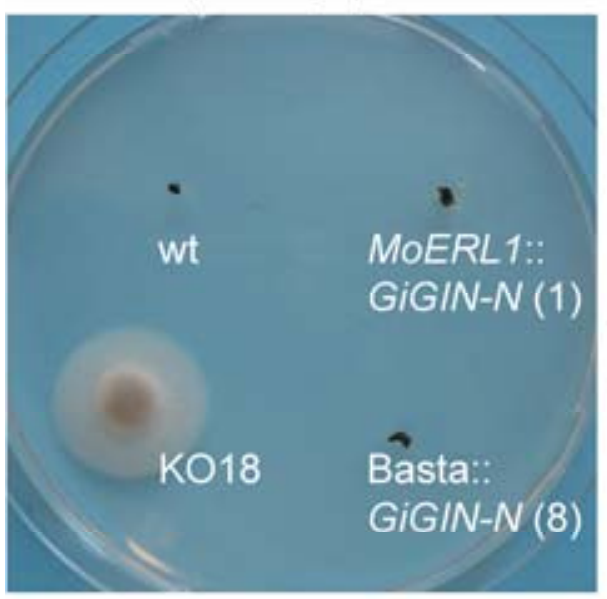

CM-Hygromycin

(240mg/l)

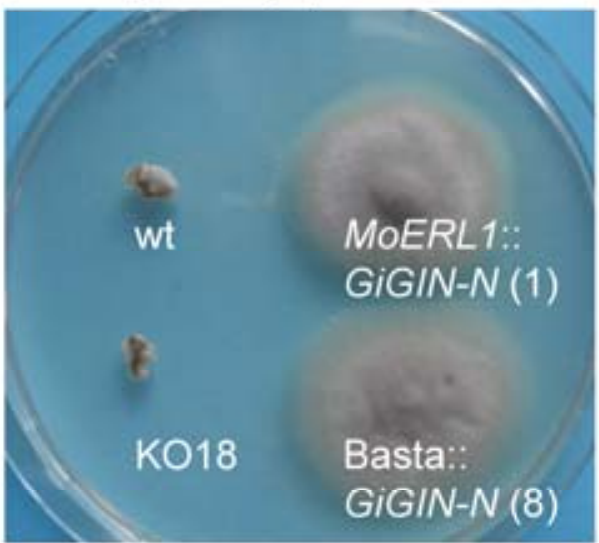

\section{B}

\section{ERL1::}

GiGIN-N

Basta::

GiGIN-N

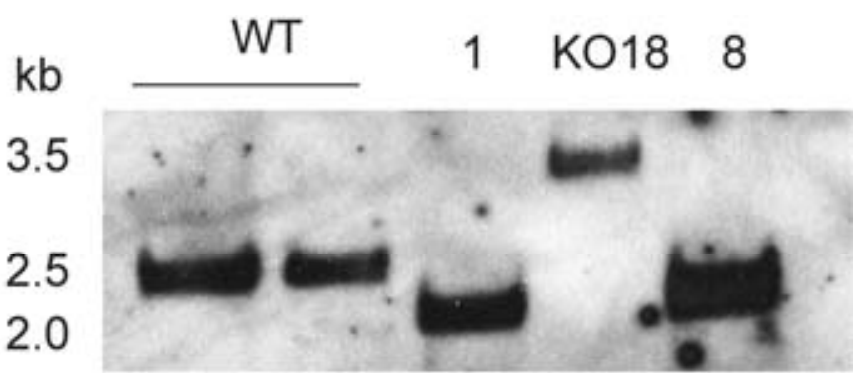

C
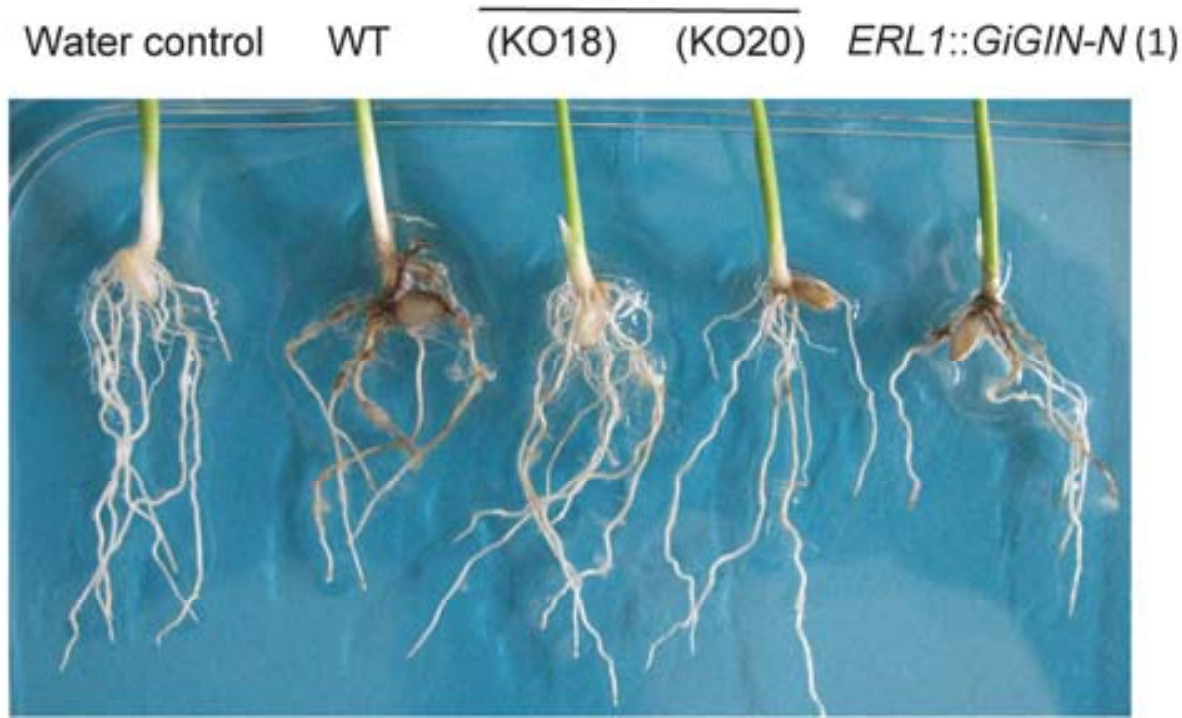

Fig. 7. Glomus intraradices Gin- $N$ complements the defect of $\Delta$ erll strains. The upstream region of GIN1 encoding the mature amino terminus was used to complement the Aerl1 strain or to substitute ERL1 in locus. A, Two strains in which GIN-N was inserted in the location of ERL1 were obtained. Strains were phenotypically tested on complete medium (CM), on CM with hygromycin, and on Sweigard-BASTA (Sweigard et al. 1997). Strains expressing GIN-Nhygromycin cassette in the locus of ERL1 were resistant to hygromycin, in contrast to the wild type (Guy11) and the 4 erl1 strain KO18. In contrast, only KO18 is resistant to BASTA. B, Southern blot of these strains after digestion with XhoI probed with the upstream region of ERL1. Both strains containing GIN-N in the ERL1 locus showed the expected reduction in size of the probed fragment, while the Aerll strain KO18 showed the expected increase in size. C, Strain ERL1::GIN-N (1) was used to inoculate rice roots. In contrast to the Aerl1 strain KO18, which produced less root browning after 15 days, the strain expressing GIN-N under the control of the ERL1 promoter showed similar browning symptoms to the wild-type strain. 

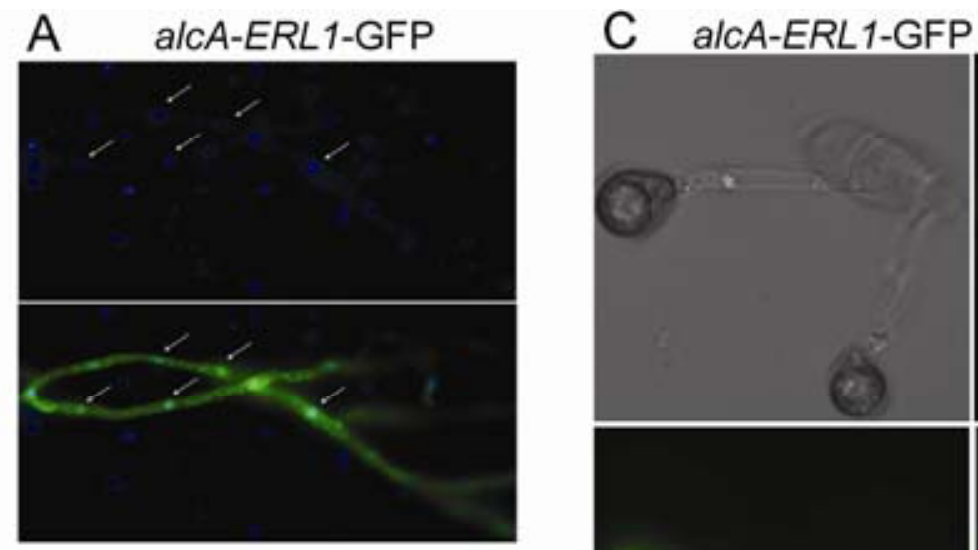

B
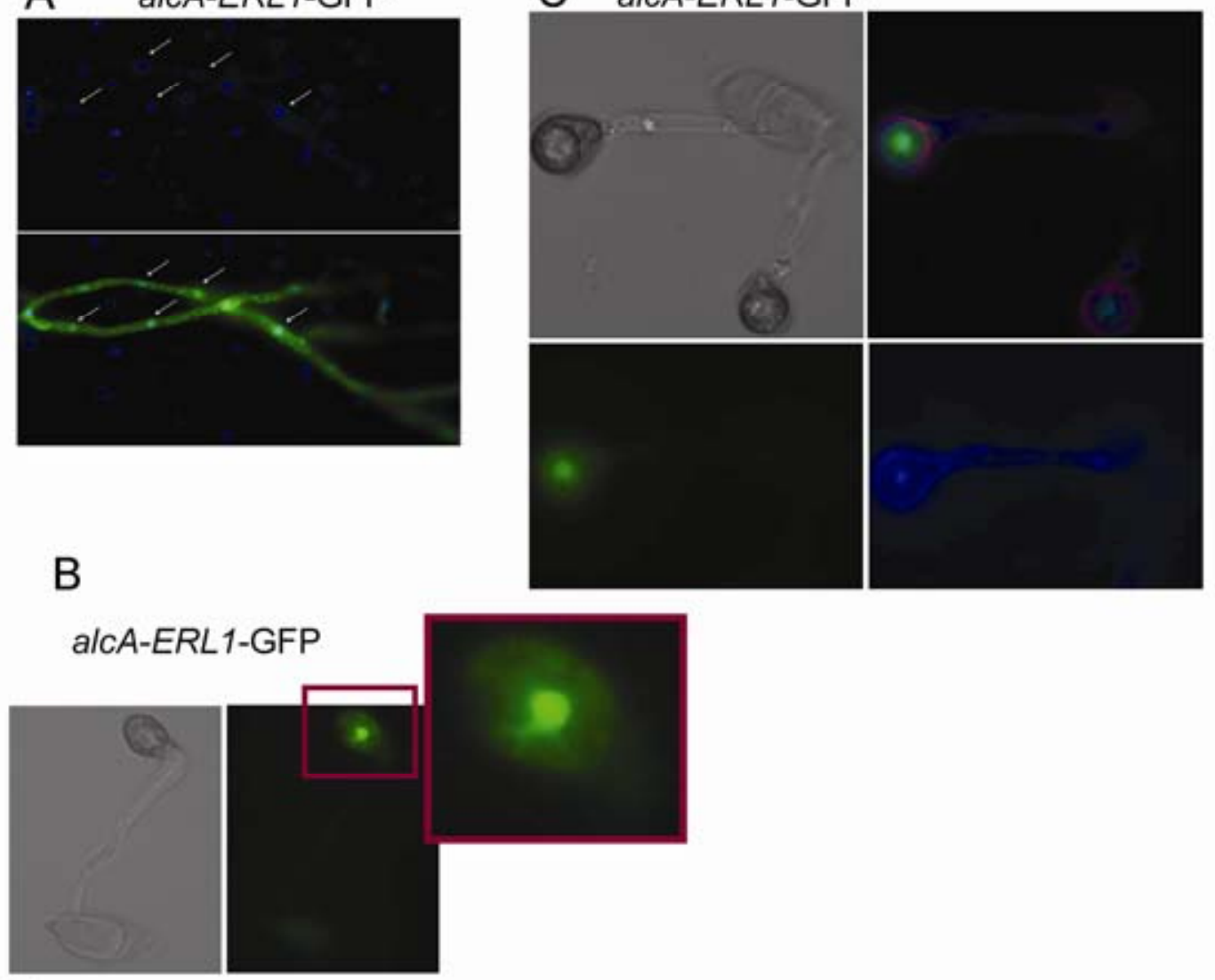

D
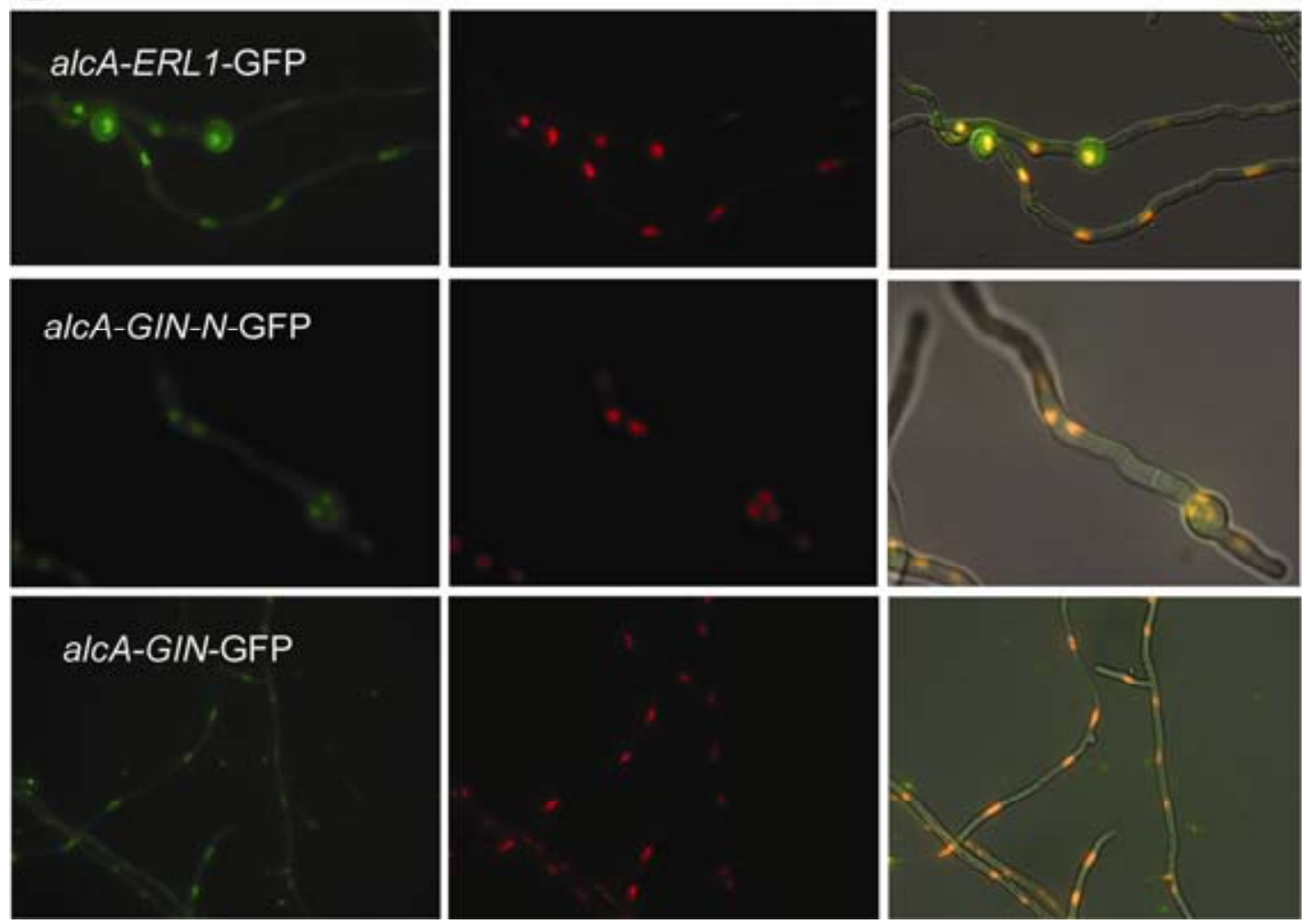

Fig. 8. Erl1 localizes to the nucleus. An expression cassette containing the ERL1 gene fused to green fluorescent protein (GFP) was expressed under the control of the alcA promoter in Magnaporthe oryzae. A, ERL1-GFP fusion protein could be observed in the nuclei of vegetative hyphae as well as B and C, in the nucleus of the appressorium. However, the level of expression was very high and endoplasmatic reticulum expression could also be observed. C, Colocalization of nuclei stained with 4',6-diamidino-2-phenylindole and Erl1-GFP in two appressoria. D, The alcA-ERL1-GFP cassette as well as two other cassettes expressing Gin-N or the full Gin protein fused to GFP (also under the control of the alcA promoter) were used to transform Aspergillus nidulans. Nuclear localization of the three fusion proteins could be confirmed by coexpression of the pJH19 plasmid expressing the fusion protein StuA-DsRED. The hyphae did not show any morphological or polarity alteration with respect to wild-type hyphae (not shown). 
A
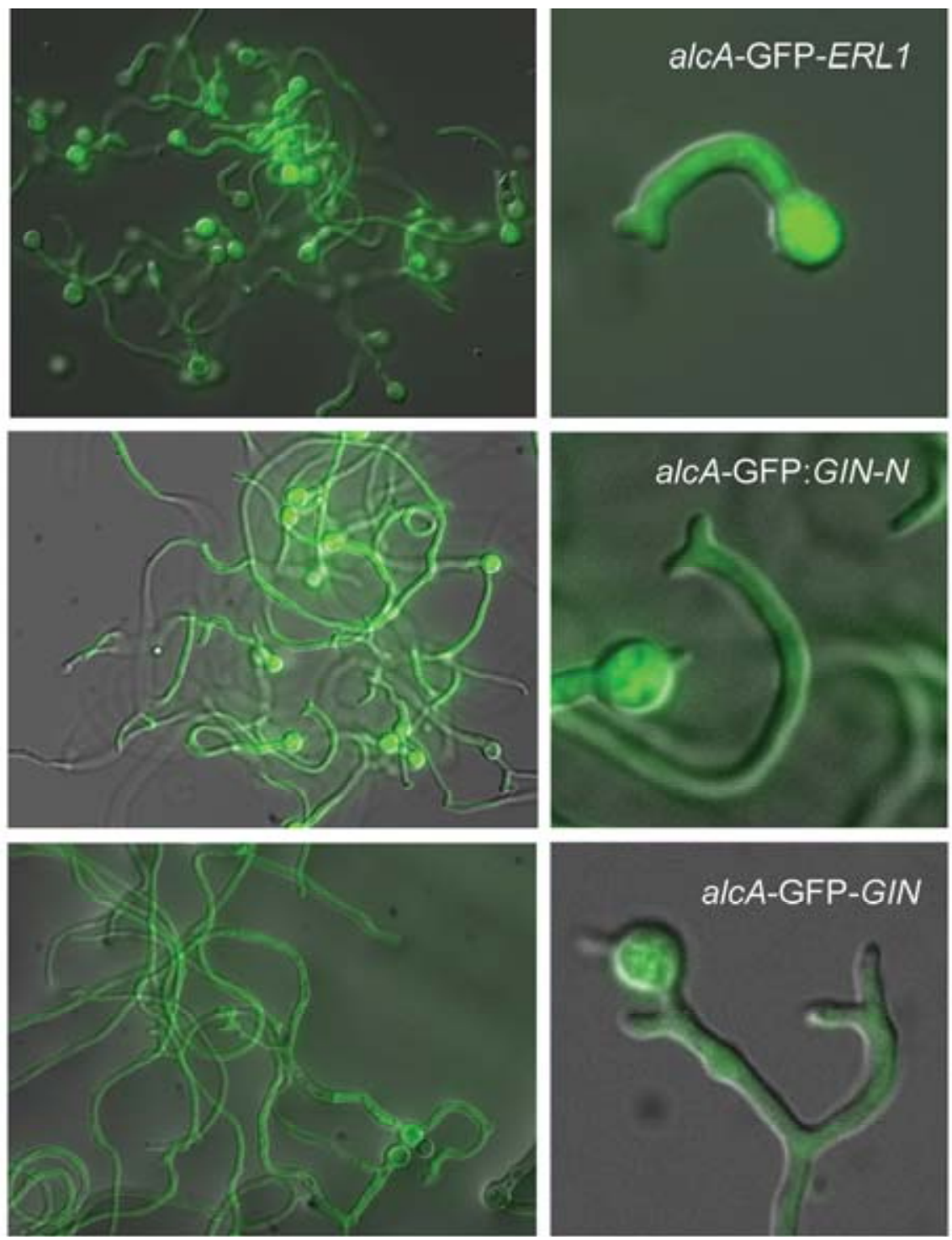

B

Gin

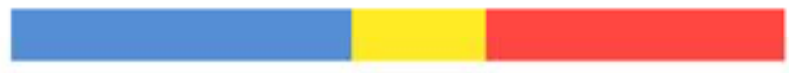

\section{Gin-N}

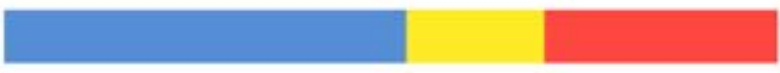

Erl1

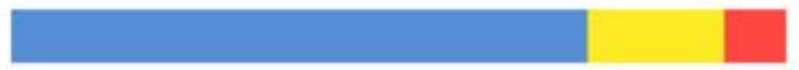

WT

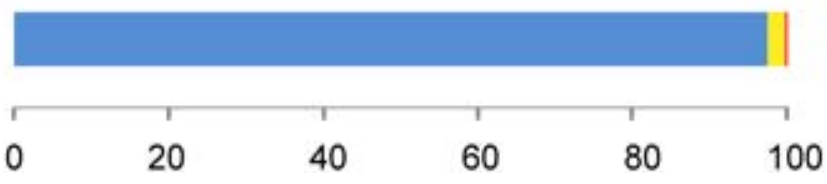

MP

BP

DB
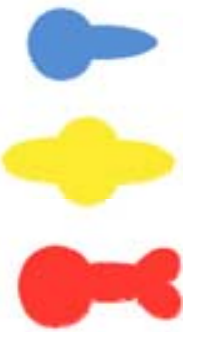

Fig. 9. Cytoplasmic localization of Er11, Gin-N, or Gin proteins induces alterations in cell polarity in Aspergillus nidulans. Green fluorescent protein (GFP) amino-terminal tagged version of Erl1, Gin-N, and Gin full protein were transformed in A. nidulans. A, All three transformed strains showed cytoplasmic localization of the fusion proteins and changes in polarity. Hyphae grew in a meandering way, and often, the hyphal tip showed dichotomous branching. B, Quantification of hyphal morphological changes in young germlings among the wild-type and the transformed strains. More than 300 germinated conidia were counted for each strain, and the experiment was repeated three times. $\mathrm{MP}=$ monopolar; $\mathrm{BP}=$ bipolar; and $\mathrm{DB}=$ dichotomous branching. The wild-type strain exhibited more than $90 \%$ monopolar germination. This percentage was significantly reduced in all transformed strains that had a higher proportion of bipolar germinated germlings and dichotomous branched germlings. This phenotype was especially severe in the A. nidulans strain expressing the fusion protein Gin-GFP. 
link connecting fungal differentiation to ROS (reactive oxygen species). ROS have been shown to be essential components determining hyphal development in several filamentous fungi (Hansberg and Aguirre 1990; Lara-Ortíz et al. 2003; Michán et al. 2003). More importantly, ROS have been shown to be critical to maintain fungal-plant interactions, including the $M$. oryzae-rice pathogen system (Egan et al. 2007; Takemoto et al. 2006; Tanaka et al. 2006, 2008). However, only recently specific GTPases have been shown to be involved in the generation of ROS by transcriptional or post-transcriptional control of NADPH oxidases (Chen and Dickmann 2005; Chen et al. 2008; Rolke and Tudzynski 2008; Takemoto et al. 2006). Interestingly, the phenotype of cytoplasmic localized Erl1 in A. nidulans resembles the phenotype observed by Semighini and Harris (2008) when they deleted NoxR, the regulator of NoxA, or treated the hyphae with DPI (diphenyleneiodonium chloride), an inhibitor of ROS production. This suggests that cytoplasmic localization of Erl1 could interfere with the production of ROS required for the establishment of polarity. In $M$. oryzae, the involvement of NADPH oxidases have been directly related to polarity determination (Egan et al. 2007). Recently, Chen and associates (2008) have shown that the GTPase Rac1 in M. oryzae controls the production of ROS by interacting with Nox1 and Nox2 (NADPH oxidases). The expression in $M$. oryzae of the amino-terminally labeled versions of Erl1 will help to elucidate whether a similar phenotype in which polarity is distorted is observed and whether Erl1 might be involved in ROS regulation.

In summary, we have identified and characterized the first Era-like GTPase in filamentous fungi. The protein is required for full root virulence in $M$. oryzae and hyphal growth in planta. More interestingly, the virulence defect can be complemented by the orthologous protein from the mutualistic symbiont $G$. intraradices. This supports and extends the hypothesis of conserved genetic features underlying plant colonization strategies among different fungi.

\section{MATERIALS AND METHODS}

\section{Fungal strains, culture conditions, and DNA analysis.}

Wild type (Guy11) and mutant strains of M. oryzae are stored in the laboratory of N. Requena, University of Karlsruhe. Standard procedures of $M$. oryzae growth, maintenance, nucleic acid extraction, and transformation were performed as described previously (Villalba et al. 2008). BASTA transformants were selected in SW-Basta (Sweigard et al. 1997): yeast nitrogen base at $1.6 \mathrm{~g} /$ liter without amino-acid, asparagine at $2 \mathrm{~g} /$ liter, $\mathrm{NH}_{4} \mathrm{NO}_{3}$ at $1 \mathrm{~g} /$ liter, glucose at $1 \mathrm{~g} / \mathrm{liter}$, and glufosinate at $0.040 \mathrm{~g} /$ liter (BASTA, Bayer Crop Sciences, Monheim, Germany).

A. nidulans RMS011 (Stringer et al. 1991) was used for the heterologous expression of Erl1- and Gin1-tagged proteins. Standard A. nidulans transformation protocols were used (Yelton et al. 1984).

\section{Targeted gene disruption.}

Two independent deletion strains were obtained in which ERL1 was replaced by the BASTA resistance cassette. The gene replacement cassette was created according to Kämper (2004). The deletion construct was made after amplifying the upstream (approximately $1.5 \mathrm{~kb}$ ) and downstream (approximately $1.5 \mathrm{~kb}$ ) regions of ERL1 by PCR, using genomic DNA (100 ng) as template. Primer pairs KOupF1/KOupR1-SfiA and KOdownF1-SfiB/KOdownR1 and $0.5 \mathrm{U}$ of DNA polymerase Expand High Fidelity (Roche Applied Science, Mannheim, Germany). The primers are listed in Supplementary Table S1. Purified amplicons were digested by SfiI, whose restriction sites are present in primers KOupR1-SfiA and KOdownF1SfiB. BASTA cassettes (Sweigard et al. 1997) conferring resistance to BASTA were excised from plasmid pFV9 (pB2KS vectors carrying a BASTA cassette flanked by SfiIa and Sfilb sites (Villalba et al. 2008). Following ligation, the genereplacement cassette (approximately $3.5 \mathrm{~kb}$ ) was obtained by PCR amplification using primers KOupF2 and KOdownR2 and was used for protoplast transformation.

\section{Functional complementation assays.}

A genomic cosmid library of $M$. oryzae was screened using the ERL1 gene as a probe. The library was described by Dioh and associates (1997). A cosmid called 9N9 was identified that contained the ERL1 gene in its genomic background. This cosmid was used to transform the ERL1 deletion strains KO18 and $\mathrm{KO} 20$ by protoplast transformation. Transformants were selected on hygromycin-containing plates as described by Villalba and associates (2008). To complement the ERL1 deletion strain KO18 with the Gin-N gene, a deletion construct was made by amplifying, on $G$. intraradices germinated spore cDNA, the $G I N-N$ sequence flanked by SfiA and SfiB sites. Primers GinN-SfiA and Gin-N-SfiBwere used. The amplicon was Sfi-digested and was cloned into the BASTA deletion cassette described above. The resulting plasmid had $G I N-N$ replacing the BASTA cassette and was flanked by the upstream and downstream ERL1 sequences. A hygromycin resistance cassette (from plasmid pFV8, Villalba et al. 2008) was introduced by blunt-end cloning in the DraI site at the beginning of the ERL1 downstream sequence. The whole replacement cassette containing the ERL1 upstream sequence, the GinN-hygromycin cassette, and ERL1 downstream sequence was PCRamplified using primers CACC-KOupF2 and KOdownR2 and was cloned into the pENTR/D-TOPO vector from Invitrogen, Karlsruhe, Germany). The cassette was introduced into the binary destination vector pKGW.0 (Karimi et al. 2002) and was transformed into the wild type (Guy11) or the ERL1-deletion strain $\mathrm{KO} 18$ by Agrobacterium tumefaciens-mediated transformation (using the AGL1 strain) as described (Betts et al. 2007).

\section{Assays for infection-related morphogenesis.}

Conidia were collected from agar plates using $0.25 \%$ gelatin solution and were spotted on the surface of Parafilm stripes (Parafilm, Pechiney Plastic Packaging Co., Chicago) or on the hydrophobic side of bioFOIL membranes (bioFoil25 (hydrophob-hydrophil), Sartorius, Goettingen, Germany). A 100- $\mu 1$ drop of conidial suspension containing $10^{4}$ spores was incubated at $24^{\circ} \mathrm{C}$ in moist chambers. Germination and appressorium development were scored for 300 conidia at 16 and $24 \mathrm{~h}$ after inoculation. Appressorium-mediated penetration of sterile onion epidermis (Allium cepa) and detached rice leaves was assayed by placing $10^{4}$ conidia in gelatin solution onto $1-\mathrm{cm}^{2}$ sections of onion epidermis or rice leaves. Appressoria formation and cell colonization of onion cells were recorded at 24 and $48 \mathrm{~h}$ after inoculation using a Zeiss Axioimager 21 fluorescence microscope (Zeiss, Oberkochen, Germany). Leaf infection was recorded 5 days after inoculation. Infection of wounded rice leaves was performed by inoculating abraded leaves with their cuticula removed, using a sterile needle and scored after 5 days.

\section{Root infection assays.}

The plant material used was rice (Oryza sativa L.) cultivar Nipponbare (subsp. japonica). Root infection was as described by Dufresne and Osbourn (2001). Briefly, rice plants were grown in glass tubes $(20 \mathrm{~cm}$ long, $3 \mathrm{~cm}$ diameter) filled with $50 \mathrm{ml}$ of sterile calcinated clay. Each tube containing one plant 
received $20 \mathrm{ml}$ of Yoshida nutrient solution (Yoshida et al. 1976). Five mycelial plugs of $0.5 \mathrm{~cm}^{2}$ were used to inoculate each tube. Mock-inoculated tubes received sterile agar plugs. Five replicate tubes were set up for each treatment. Root infection experiments were repeated five times for all root experiments performed. Tubes were closed with glass lids that allow gas exchange. Plants were grown in a phytochamber at $25^{\circ} \mathrm{C}$ (photoperiod of $16 \mathrm{~h}$ light $\left[62.5 \mu \mathrm{E} \mathrm{m}^{-2} \mathrm{~s}^{-1}\right]$ and $8 \mathrm{~h}$ dark) for 2 to 3 weeks. The seedling roots were then washed in water and were examined for root browning. Root browning (extension and intensity) was scored using a rating system from 0 (no lesions) to 5 (root fully dark brown). Five different observers scored each treatment and experiment replicate independently, and results were expressed as percentage of root browning in Table 1. Fungal colonization of roots by GFP-tagged strains was evaluated with a Zeiss Axiophot fluorescence microscope (Zeiss).

\section{GFP tagging for protein localization.}

Carboxy-terminal GFP labeling of proteins was carried out using the destination vector pMT-sGFP (Töws et al. 2004). cDNAs encoding Erl1, Gin-N, and Gin were PCR amplified with CACC forward primers and cloned into the pENTR/DTOPO vector (Invitrogen). They were recombined into the destination vector using the GATEWAY system (Invitrogen). To create an N-terminal GFP fusion, labeling was performed by cloning the corresponding cDNAs with AspI and $P a c \mathrm{I}$ restriction sites amplified by PCR into the corresponding sites of the pCMB17apx vector (Efimov et al. 2006).

\section{DsRED labeling of nuclei in $A$. nidulans.}

For the visualization of nuclei in A. nidulans, RMS011 was transformed with the plasmid pJH19 expressing the nuclear localized protein StuA fused to the DsRED reporter under the control of the gpd promoter (Töws et al. 2004).

\section{ACKNOWLEDGMENTS}

We thank our colleagues J. Kämper and R. Fischer for critical reading of the manuscript. This work was supported by the German Science Foundation (DFG) Re-1556/3-1 and Re-1556/4-1. N. Requena is supported by the Heisenberg program from the DFG.

\section{LITERATURE CITED}

Ahnn, J., March, P. E., Takiff, H. E., and Inouye, M. 1986.A GTP-binding protein of Escherichia coli has homology to yeast RAS proteins. Proc. Natl. Acad. Sci. U.S.A. 83:8849-53.

Akiyama, T., Gohda, J., Shibata, S., Nomura, Y., Azuma, S., Ohmori, Y., Sugano, S., Arai, H., Yamamoto, T., and Inoue J. 2001.Mammalian homologue of E. coli Ras-like GTPase (ERA) is a possible apoptosis regulator with RNA binding activity. Genes Cells 6:987-1001.

Betts, M. F., Tucker, S. L., Galadima, N., Meng, Y., Patel, G., Li, L., Donofrio, N., Floyd, A., Nolin, S., Brown, D., Mandel, M. A., Mitchell, T. K., Xu, J. R., Dean, R. A., Farman, M. L., and Orbach, M. J. 2007. Development of a high throughput transformation system for insertional mutagenesis in Magnaporthe oryzae. Fungal Genet. Biol. 44:10351049.

Bhambra, G. K., Wang, Z. Y., Soanes, D. M., Wakley, G. E., and Talbot, N. J. 2006.Peroxisomal carnitine acetyl transferase is required for elaboration of penetration hyphae during plant infection by Magnaporthe grisea. Mol. Microbiol.61:46-60.

Cabedo, H., Macián, F., Villarroya, M., Escudero, J. C., Martínez-Vicente, M., Knecht, E., and Armengod, M. E. 1999.The Escherichia coli trmE (mnmE) gene, involved in tRNA modification, codes for an evolutionarily conserved GTPase with unusual biochemical properties. EMBO (Eur. Mol. Biol. Organ.) J. 18:7063-7076.

Caldon, C. E., and March, P. E. 2003.Function of the universally conserved bacterial GTPases. Curr. Opin. Microbiol. 6:135-139.

Chen, C., and Dickman, M. B. 2005. Proline suppresses apoptosis in the fungal pathogen Colletotrichum trifolii. Proc. Natl. Acad. Sci. U.S.A. $102: 3459-3464$
Chen, C., Ha, Y. S., Min, J. Y., Memmott, S. D., and Dickman, M. B. 2006. $\mathrm{Cdc} 42$ is required for proper growth and development in the fungal pathogen Colletotrichum trifolii. Eukaryotic Cell 5:155-166.

Chen, J., Zheng, W., Zheng, S., Zhang, D., Sang, W., Chen, X., Li, G., Lu, G., and Wang, Z. 2008. Rac1 is required for pathogenicity and Chm1dependent conidiogenesis in rice fungal pathogen Magnaporthe grisea. PLoS Pathog.4:e1000202. Published online.

Colby, G., Wu, M., and Tzagoloff, A. 1998. MTO1 codes for a mitochondrial protein required for respiration in paromomycin-resistant mutants of Saccharomyces cerevisiae. J. Biol. Chem. 273:27945-27952.

de Jong, J. C., McCormack, B. J., Smirnoff, N. and Talbot, N. J. 1997. Glycerol generates turgor in rice blast. Nature 389:244.

Dean, R. A., Talbot, N.J., Ebbole, D. J., Farman, M. L., Mitchell, T. K., Orbach, M. J., Thon, M., Kulkarni, R., Xu, J. R., Pan, H., Read, N. D., Lee ,Y. H., Carbone, I., Brown, D., Oh ,Y. Y., Donofrio, N., Jeong, J. S., Soanes, D. M., Djonovic, S., Kolomiets, E., Rehmeyer, C., Li,W., Harding, M., Kim, S., Lebrun, M. H., Bohnert, H., Coughlan, S., Butler, J., Calvo, S., Ma, L. J., Nicol, R., Purcell, S., Nusbaum, C., Galagan, J. E., and Birren, B. W. 2005. The genome sequence of the rice blast fungus Magnaporthe grisea. Nature 434:980-986.

Decoster, E., Vassal, A., and Faye, G. 1993.MSS1, a nuclear-encoded mitochondrial GTPase involved in the expression of COX1 subunit of cytochrome c oxidase. J. Mol. Biol. 232:79-88.

Dioh, W., Tharreau, D., and Lebrun, M. H. 1997. RAPD-based screening of genomic libraries for positional cloning. Nucleic Acids Res. 25:5130-5131.

Dufresne, M., and Osbourn, A. E. 2001.Definition of tissue-specific and general requirements for plant infection in a phytopathogenic fungus. Mol. Plant-Microbe Interact. 14:300-307.

Efimov, V., J. Zhang, and X. Xiang. 2006. CLIP-170 homologue and NUDE play overlapping roles in NUDF localization in Aspergillus nidulans. Mol. Biol. Cell 17:2021-2034.

Egan, M. J., Wang, Z. Y., Jones, M. A., Smirnoff, N., and Talbot, N. J. 2007. Generation of reactive oxygen species by fungal NADPH oxidases is required for rice blast disease. Proc. Natl. Acad. Sci. U.S.A. 104:11772-11777.

Genre, A., Chabaud, M., Faccio, A., Barker, D. G., and Bonfante, P. 2008. Prepenetration apparatus assembly precedes and predicts the colonization patterns of arbuscular mycorrhizal fungi within the root cortex of both Medicago truncatula and Daucus carota. Plant Cell. 20:14071420

Genre, A., Ortu, G., Bertoldo, C., Martino, E., and Bonfante, P. 2009. Biotic and abiotic stimulation of root epidermal cells reveals common and specific responses to arbuscular mycorrhizal fungi. Plant Physiol. 149:1424-1434.

Gong, S., Ma, Z., and Foster, J. W. 2004. The Era-like GTPase TrmE conditionally activates gadE and glutamate-dependent acid resistance in Escherichia coli. Mol. Microbiol. 54:948-961.

Güimil, S., Chang, H. S., Zhu, T., Sesma, A., Osbourn, A., Roux, C., Ioannidis, V., Oakeley, E. J., Docquier, M., Descombes, P., Briggs, S. P., and Paszkowski, U. 2005. Comparative transcriptomics of rice reveals an ancient pattern of response to microbial colonization. Proc. Natl. Acad. Sci. U.S.A. 102:8066-8070.

Hansberg, W., and Aguirre, J. 1990. Hyperoxidant states cause microbial cell differentiation by cell isolation from dioxygen. J. Theor. Biol. 142:201-221.

Kämper, J., Kahmann, R., Bölker, M., Ma, L. J., Brefort, T., Saville, B. J., , Banuett, F., Kronstad, J. W., Gold, S. E., Müller, O., Perlin, M. H., Wösten, H. A., de Vries, R., Ruiz-Herrera, J., Reynaga-Peña, C. G., Snetselaar, K., McCann, M., Pérez-Martín, J., Feldbrügge, M. Basse, C. W., Steinberg, G., Ibeas, J. I., Holloman, W., Guzman, P., Farman, M., Stajich, J. E., Sentandreu, R., González-Prieto, J. M., Kennell, J. C., Molina, L., Schirawski, J., Mendoza-Mendoza, A., Greilinger, D., Münch, K., Rössel, N., Scherer, M., Vranes, M., Ladendorf, O., Vincon, V., Fuchs, U., Sandrock, B., Meng, S., Ho, E. C., Cahill, M. J., Boyce, K. J., Klose, J., Klosterman, S. J., Deelstra, H. J., Ortiz-Castellanos, L., Li, W., Sanchez-Alonso, P., Schreier, P. H., Häuser-Hahn, I., Vaupel, M., Koopmann, E., Friedrich, G., Voss, H., Schlüter, T., Margolis, J., Platt, D., Swimmer, C., Gnirke, A., Chen, F., Vysotskaia, V., Mannhaupt, G., Güldener, U., Münsterkötter, M., Haase, D., Oesterheld, M., Mewes, H. W., Mauceli, E. W., DeCaprio, D., Wade, C. M., Butler, J., Young, S., Jaffe, D. B., Calvo, S., Nusbaum, C., Galagan, J., and Birren, B. W. 2006. Insights from the genome of the biotrophic fungal plant pathogen Ustilago maydis. Nature 444:97-101.

Kankanala, P., Czymmek, K., and Valent, B. 2007. Role for rice membrane dynamics and plasmodesmata during biotrophic invasion by the blast fungus. Plant Cell 19:706-724.

Karimi, M., Inzé, D., and Depicker, A. 2002.GATEWAY(TM) vectors for Agrobacterium-mediated plant transformation. Trends Plant Sci.7:193195. 
Lara-Ortíz, T., Riveros-Rosas, H., and Aguirre, J. 2003. Reactive oxygen species generated by microbial NADPH oxidase NoxA regulate sexual development in Aspergillus nidulans. Mol. Microbiol. 50:12411255.

Mahlert, M., Leveleki, L., Hlubek, A., Sandrock, B. and Bolker, M. 2006. Rac1 and $\mathrm{Cdc} 42$ regulate hyphal growth and cytokinesis in the dimorphic fungus Ustilago maydis. Mol. Microbiol. 59:567-578.

Marchler-Bauer, A., Anderson, J. B., Derbyshire, M. K., DeWeese-Scott, C., Gonzales, N. R., Gwadz, M., Hao, L., He, S., Hurwitz, D. I., Jackson, J. D., Ke, Z., Krylov, D., Lanczycki, C. J., Liebert, C. A., Liu, C., Lu, F., Lu, S., Marchler, G. H., Mullokandov, M., Song, J. S., Thanki, N., Yamashita, R. A., Yin, J. J., Zhang, D., and Bryant, S. H. 2007. CDD: A conserved domain database for interactive domain family analysis. Nucleic Acids Res. 35:237-240.

Martin, F., Aerts, A., Ahrén, D., Brun, A., Danchin, E. G., Duchaussoy, F., Gibon, J., Kohler, A., Lindquist, E., Pereda, V., Salamov, A., Shapiro, H. J., Wuyts, J., Blaudez, D., Buée, M., Brokstein, P., Canbäck, B., Cohen, D., Courty, P. E., Coutinho, P. M., Delaruelle, C., Detter, J. C., Deveau, A., DiFazio, S., Duplessis, S., Fraissinet-Tachet, L., Lucic, E., Frey-Klett, P., Fourrey, C., Feussner, I., Gay, G., Grimwood, J., Hoegger, P. J., Jain, P., Kilaru, S., Labbé „J., Lin, Y. C., Legué, V., Le Tacon, F., Marmeisse, R., Melayah, D., Montanini, B., Muratet, M., Nehls, U., Niculita-Hirzel, H., Oudot-Le Secq, M. P., Peter, M., Quesneville, H., Rajashekar, B., Reich, M., Rouhier, N., Schmutz, J., Yin, T., Chalot, M., Henrissat, B., Kües, U., Lucas, S., Van de Peer, Y., Podila, G. K., Polle, A., Pukkila, P. J., Richardson, P. M., Rouzé, P., Sanders, I. R., Stajich, J. E., Tunlid, A., Tuskan, G., and Grigoriev, I. V. 2008. The genome of Laccaria bicolor provides insights into mycorrhizal symbiosis. Nature 452:88-92.

Michán, S., Lledías, F., and Hansberg, W. 2003.Asexual development is increased in Neurospora crassa cat-3-null mutant strains. Eukaryot. Cell 2:798-808.

Mittenhuber, G. 2001. Comparative genomics of prokaryotic GTP-binding proteins (the Era, Obg, EngA, ThdF (TrmE), YchF and YihA families) and their relationship to eukaryotic GTP-binding proteins (the DRG, ARF, RAB, RAN, RAS and RHO families). J. Mol. Microbiol. Biotechnol.3:21-35.

Mosquera, G., Giraldo, M. C., Hyun Khang, C., Coughlan, S., and Valent, B. 2009. Interaction transcriptome analysis identifies Magnaporthe oryzae BAS1-4 as biotrophy-associated secreted proteins in rice blast disease. Plant Cell 21:1273-1290.

Paulus, H. 2000.Protein splicing and related forms of protein autoprocessing. Annu. Rev. Biochem. 69:447-496.

Requena, N., Mann, P., Hampp, R., and Franken, P. 2002. Early developmentally regulated genes in the arbuscular mycorrhizal fungus Glomus mosseae: Identification of GmGIN1 a novel gene with homology to the C-terminus of metazoan hedgehog proteins. Plant Soil 244:129-139.

Requena, N., Serrano, E., Ocón, A., and Breuninger, M. 2007. Plant signals and fungal perception during arbuscular mycorrhiza establishment. Phytochemistry68:33-40.

Rolke, Y., and Tudzynski, P. 2008. The small GTPase Rac and the p21activated kinase Cla4 in Claviceps purpurea: Interaction and impact on polarity, development and pathogenicity. Mol. Microbiol. 68:405-423.

Roslan, H. A., Salter, M. G., Wood, C. D., White, M. R., Croft, K. P., Robson, F., Coupland, G., Doonan, J., Laufs, P., Tomsett, A. B., and Caddick, M. X. 2001.Characterization of the ethanol-inducible alc geneexpression system in Arabidopsis thaliana. Plant J. 28:225-235.
Semighini, C. P. and Harris, S. D. 2008. Regulation of apical dominance in Aspergillus nidulans hyphae by reactive oxygen species. Genetics 179:1919-1932.

Sesma, A., and Osbourn, A. E. 2004. The rice leaf blast pathogen undergoes developmental processes typical of root-infecting fungi. Nature 431:582-586.

Soanes, D. M., Alam, I., Cornell, M., Wong, H. M., Hedeler, C., Paton, N. W., Rattray, M., Hubbard, S. J., Oliver, S. G., and Talbot, N. J. 2008 Comparative genome analysis of filamentous fungi reveals gene family expansions associated with fungal pathogenesis. PLoS ONE 3:e2300.

Stringer, M. A., Dean, R. A., Sewall, T. C., and Timberlake, W. E. 1991. Rodletless, a new Aspergillus developmental mutant induced by directed gene inactivation. Genes Dev. 5:1161-1171.

Sweigard, J. A., Chumley, F., Carroll, A. M., Farrall, L., and Valent, B. 1997. A series of vectors for fungal transformation. Fungal Genet. Newsl. 44:52-53.

Talbot, N. J. 2003. On the trail of a cereal killer: Exploring the biology of Magnaporthe grisea. Ann. Rev. Microbiol. 57:177-202.

Takemoto, D., Tanaka, A., and Scott, B. 2006. A p67Phox-like regulator is recruited to control hyphal branching in a fungal-grass mutualistic symbiosis. Plant Cell 18:2807-2821.

Tanaka, A., Christensen, M. J., Takemoto, D., Park, P., and Scott. B. 2006. Reactive oxygen species play a role in regulating a fungus-perennial ryegrass mutualistic interaction. Plant Cell18:1052-1066.

Tanaka, A., Takemoto, D., Hyon, G. S., Park, P., and Scott, B. 2008. NoxA activation by the small GTPase RacA is required to maintain a mutualistic symbiotic association between Epichloë festucae and perennial ryegrass. Mol. Microbiol. 68:1165-1178.

Thompson, J. D., Higgins, D. G. and Gibson, T. J. 1994. CLUSTAL W: Improving the sensitivity of progressive multiple sequence alignment through sequence weighting, position specific gap penalties and weight matrix choice. Nucleic Acids Res22:4673-4680.

Töws, M. W., Warmbold, J., Konzack, S., Rischitor, P., Veith, D., Vienken, K., Vinuesa, C., Wei, H., and Fischer, R. 2004. Establishment of mRFP1 as a fluorescent marker in Aspergillus nidulans and construction of expression vectors for high-throughput protein tagging using recombination in vitro (GATEWAY). Curr. Genet. 45:383-389.

Villalba, F., Collemare, J., Landraud, P., Lambou, K., Brozek, V., Cirer, B., Morin, D., Bruel, C., Beffa, R., and Lebrun M. H. 2008. Improved gene targeting in Magnaporthe grisea by inactivation of MgKU80 required for non-homologous end joining. Fungal Genet. Biol. 45:68-75.

Yelton, M. M., Hamer, J. E., and Timberlake, W. E. 1984. Transformation of Aspergillus nidulans by using a trpC plasmid. Proc. Natl. Acad. Sci. U.S.A. 81:1470-1474.

Yoshida, S., Forno, D. A., Cook, J. H. and Gomez, K. A. 1976. Pages 6166 in: Laboratory Manual for Physiological Studies of Rice. International Rice Research Institute, Philippines.

Wang, G. Y., and Keasling, J. D. 2002.Amplification of HMG-CoA reductase production enhances carotenoid accumulation in Neurospora crassa. Metab. Eng. 4:193-201.

Williams, C. L. 2003.The polybasic region of Ras and Rho family small GTPases: A regulator of protein interactions and membrane association and a site of nuclear localization signal sequences. Cell Signal 15:10711080

Zheng, W., Chen, J., Liu, W., Zheng, S., Zhou, J., Lu, G., and Wang, Z. 2007. A Rho3 homolog is essential for appressorium development and pathogenicity of Magnaporthe grisea. Eukaryot. Cell 6:2240-2250. 